\title{
AVES ACUÁTICAS Y PAISAJE FLUVIAL EN LAS RIBERAS DE LOS RÍOS EBRO, TAJO Y JARAMA. CARACTERÍSTICAS GENERALES
}

\author{
Pedro MOLINA HOLGADO \\ Departamento de Geografía. Universidad Autónoma de Madrid \\ Ana Belén BERROCAL MENÁRGUEZ \\ Departamento de Ingeniería Civil: Ordenación del Territorio, Urbanismo y \\ Medio Ambiente. Universidad Politécnica de Madrid
}

Recibido: 08/04/2011

Devuelto: 13/05/2011

Aceptado: 19/07/2011

RESUMEN: Los grandes ríos ibéricos son ámbitos de interés para las aves acuáticas. Su importancia es especialmente destacada en ciclos climáticos secos, cuando la mayor parte de las zonas húmedas naturales o seminaturales permanecen secas o registran bajos niveles de agua. La importancia de estos medios varía, no obstante, en relación con las características del paisaje fluvial, especialmente las referidas a la calidad del agua, funcionamiento hidráulico, naturalidad del régimen hidrológico o morfología del canal. En este artículo se analizan las características básicas de las comunidades de aves acuáticas de los ríos Ebro, Tajo y Jarama, tres cursos fluviales situados en los sectores centrales de dos grandes cuencas sedimentarias terciarias, equiparables por su potencialidad natural aunque afectados en diverso grado por distintas intervenciones antrópicas. Este análisis se basa en los resultados de censos de aves acuáticas realizados durante un ciclo anual sobre más de $64 \mathrm{~km}$ de cauces.

PALABRAS CLAVE: aves acuáticas, ríos, paisaje fluvial, Ebro, Tajo, Jarama.

WATERBIRDS AND FLUVIAL LANDSCAPES ALONG THE BANKS OF THE EBRO, TAJO AND JARAMA RIVERS. GENERAL CHARACTERISTICS

ABSTRACT: Large Iberian rivers are the major focus areas for waterbirds. Its importance is particularly strong in dry climate cycles, when the majority of semi-natural wetlands remain dry or have low water levels. The importance of these methods varies, however, regarding the characteristics of the river landscape, particularly with respect to water quality, hydraulic performance, natural hydrological regime and channel morphology. This paper discusses the basic features of aquatic bird communities of the river Ebro, Tajo and Jarama, three rivers located in the central part of two major Tertiary sedimentary basins, comparable to its natural potential but affected to varying degrees by different anthropogenic intervention. Is based on the results of waterbirds census conducted during an annual cycle over $64 \mathrm{~km}$ of channels.

KEY WORDS: waterbirds, rivers, fluvial landscape, Ebro, Tajo, Jarama. 


\section{INTRODUCCIÓN}

Los estudios dedicados a las comunidades de aves acuáticas de cursos fluviales son relativamente escasos en España, a diferencia de lo que sucede en otros países (BRAUZE, 2004; HUBÁLEK, 1999; KOFFIJBERG et AL., 2001; MASON y MACDONALD, 2000; MASON et $A L$., 2006; ROUND y MOSS, 1984; STEVENS et $A L ., 1997)$ o con otras zonas húmedas, sobre las que sí existe un extenso y actualizado repertorio de trabajos. Hasta finales de la década de 1990 los trabajos referidos a la avifauna de los ríos de España fueron muy escasos, centrándose en muchos casos en especies o grupos de aves en alguno de los cursos más importantes de la Cuenca del Tajo (BLANCO et $A L$., 1994; BLANCO et $A L ., 1995)$, en los ríos del tercio Norte peninsular (CAMPOS y LEKUONA, 1994; CUEVAS, 1997; GÁMEZ; 1994; HERNÁNDEZ y VELASCO, 1990; LEKUONA y CAMPOS, 1996) o en áreas más extensas (VELASCO, 1992; VELASCO y ALBERTO, 1993) y, en menor medida, en otros ámbitos geográficos (LEIVA et AL., 1989; MoliNA et AL., 1987). Desde entonces, sin ser un tema de análisis preferente, un buen número de tramos fluviales son habitualmente censados durante los periodos de invernada o reproducción (p.e. SEO-BIRDLIFE, 1997-1999; SEO-ARAGÓN, 1994, 1997; DEL MORAL, 2000-2003; Molina, 2004-2007; ARRATIBEL et AL., 1995, 1996) y han aparecido además algunos trabajos globales referidos a este tipo de medios (VElasco, 2009; Cuevas et AL.; 2000a, 2000b; CANO, 2005).

De esta escasez de estudios se deriva un conocimiento fragmentario de las características y dinámica de estas comunidades de aves en la Península Ibérica, a pesar de la reconocida importancia de estos medios (ARTHINGTON et $A L ., 2010)$. A pesar también de albergar un buen número de especies sensibles (REBANE et $A L ., 1997$ ) y hábitats en muchos casos protegidos al amparo de la normativa vigente, tanto en España como en la Unión Europea (CALleJa, 2009; TORO et AL., 2009).

La densidad media de las comunidades de aves acuáticas en los ríos Ebro y Tajo-Jarama ilustra bien la importancia de estos ámbitos: el sector analizado de estos cursos puede llegar a albergar en años de sequía, como durante el bienio aquí analizado (1994-1995), hasta el 30\% del total de la población invernante de aves acuáticas en la región de Madrid, posiblemente debido a la desaparición temporal de los humedales de La Mancha como consecuencia de la ausencia continuada de precipitaciones (AMAT y FERRER, 1988). Algunos cursos ibéricos como los ríos Zújar, Ebro, Tajo o Jarama, entre otros, son además áreas de interés para la invernada de alguna aves acuáticas ( $\mathrm{Ta}$ chybaptus ruficollis, Podiceps cristatus, Phalacrocorax carbo, Anas platyrhynchos, Anas strepera) (MARTÍ y DEL MORAL, 2003). 
Por todo ello, parece de interés presentar este trabajo, cuyo objetivo es la caracterización de las comunidades de aves acuáticas de las riberas de los ríos Ebro, Tajo y Jarama en un extenso sector fluvial situado en Navarra, Aragón, Madrid y Castilla-La Mancha. Pretende además identificar las afinidades y diferencias básicas existentes entre estas comunidades y los factores causantes de las mismas, características relacionadas en gran medida con la estructura y conservación de los hábitats ribereños y fluviales. Se basa en datos propios inéditos tomados durante un ciclo anual durante los años 1994, 1995 y 1996.

\section{MATERIAL Y MÉTODOS}

Los datos utilizados para la caracterización de las comunidades de aves acuáticas se han obtenido mediante la realización de censos, cómputos globales de individuos basados en el conteo directo de todas las aves acuáticas presentes en un espacio de dimensiones conocidas durante un tiempo determinado (TELlERÍA, 1986). La zona de censo en los ríos estudiados abarca únicamente el canal de bankfull; no se han tomado datos en los humedales cercanos a los tramos fluviales prospectados (graveras, arrozales, eriales encharcados, galachos, etc.), salvo en el caso de que éstos fueran el resultado de modificaciones artificiales del canal y mantuvieran conexión estructural con el mismo.

En los ríos Tajo y Jarama se ha censado, con periodicidad mensual entre febrero de 1994 y febrero de 1995 (13 meses), un sector fluvial cuya longitud total fue de $32,8 \mathrm{~km}$, repartido en 19 subtramos (Cuadro 1), de los que 6 correspondían al Jarama $(12,5 \mathrm{~km})$ y 13 al Tajo $(20,3 \mathrm{~km})$.

En el río Ebro se ha censado hasta un máximo de 24,6 km de río. La zona de censo se encontraba repartida en un máximo de 12 subtramos, realizándose los conteos durante un ciclo de 13 meses (septiembre 1995-septiembre 1996), aunque la longitud habitual de censo varió entre 22,4 y $23,3 \mathrm{~km}$ y entre 10 y 11 tramos de censo (CUADRO 2). Los resultados de estos censos están recogidos en los cuadros 10,11 y 12 .

La elección de tramos se realizó en virtud de la facilidad de acceso a los mismos e intentando que éstos estuvieran repartidos de manera homogénea a lo largo de todo el área de estudio. Otro criterio importante a la hora de seleccionar los tramos fue su aislamiento o lejanía de zonas de concentración de aves acuáticas externas a la ribera, ya que el objetivo de estos censos ha sido conocer con la mayor precisión posible la potencialidad de los ríos estudiados para este grupo de aves. Por este motivo, se desecharon algunos 
tramos fluviales que limitaban con graveras y galachos, debido al constante trasiego y entrada de aves de estas zonas húmedas al río o viceversa.

Cuadro 1. Características de los tramos censados (Tajo-Jarama)

\begin{tabular}{clccl}
\hline$N^{o}$ & \multicolumn{1}{c}{ Denominación } & Río & $\begin{array}{c}\text { Long } \\
(\mathrm{km})\end{array}$ & \multicolumn{1}{c}{ UTM(*) } \\
\hline 1 & Puente de El Pindoque & Jarama & 2,2 & VK5359-5358-5357 \\
2 & Titulcia-escarpe & Jarama & 2 & VK5144-5145-5245-5246 \\
3 & Titulcia-Tajuña & Jarama & 1,7 & VK5042 \\
4 & Ciempozuelos & Jarama & 2 & VK4840-4841 \\
5 & Pte. Largo-Meandro del Jembleque & Jarama & 2 & VK4738-4838-4837 \\
6 & Soto de Tardío & Jarama & 1,7 & VK4635-4735-4635-4735 \\
7 & Junta de los ríos Tajo-Jarama & J.-T. & 1 & VK4431-4432 \\
8 & Puente de la autovía de Andalucía & Tajo & 0,6 & VK4332-4432 \\
9 & Valdebajares 1 & Tajo & 1,1 & VK4120-4130 \\
10 & Valdebajares 2 & Tajo & 1 & VK4029-4020 \\
11 & Valdebajares 3 & Tajo & 1 & VK4030-3930-3929 \\
12 & Puente de la Barca & Tajo & 2 & VK3838-3827 \\
13 & Puente y escarpe de Añover & Tajo & 2,5 & VK3825-3824-3724-3723-3623-3624 \\
14 & Chopera de Barciles & Tajo & 1,4 & VK3323-3223-3222-3322 \\
15 & Granja de Barciles Bajo & Tajo & 1 & VK3122-3123 \\
16 & Los Arenales & Tajo & 1,2 & VK3023-3022 \\
17 & Casa Cisneros & Tajo & 1 & VK2921-2920 \\
18 & Aceca & Tajo & 4 & VK2821-2721-2621-2520 \\
19 & Algodor & Tajo & 2,5 & VK2520-2519-2518 \\
\hline
\end{tabular}

(*) Las coordenadas UTM indican las cuadrículas en las que se sitúan los tramos.

Cuadro 2. Características de los tramos censados (Ebro)

\begin{tabular}{clcl}
\hline$N^{o}$ & \multicolumn{1}{c}{ Denominación } & $\begin{array}{c}\text { Long } \\
(\mathrm{km})\end{array}$ & \multicolumn{1}{c}{ UTM $^{(*)}$} \\
\hline 1 & Puente de Buñuel & 1,5 & XM2948-2947 \\
2 & Puente de Novillas & 1,5 & XM3344-3343 \\
3 & Meandro de Novillas & 2,4 & XM3542-3541-3641 \\
4 & Desembocadura del Arba & 1,1 & XM3938-3937 \\
5 & Gallur & 1,3 & XM4036 \\
6 & Remolinos & 1,4 & XM5132-5131-5231 \\
7 & El Burgo de Ebro & 0,8 & XM8805-8804-8904 \\
8 & Soto de Begunex & 2,4 & XM9004-9104 \\
9 & Presa de Pina & 1,5 & XM9104-9204 \\
10 & Soto de Longueras-Pina & 3,3 & YL0497-0397-0397-0396-0496-0597-0596 \\
11 & El Figueral-Mejana Baja & 4,3 & YL0694-0794-0894-0893 \\
12 & Casa de Belloque-Quinto & 3,1 & YL0991-0891-0890 \\
\hline (*) Las coordenadas UTM indican las cuadrículas en las que se sitúan los tramos.
\end{tabular}

Además de los parámetros básicos habitualmente utilizados en el estudio de comunidades, se ha considerado también los citados a continuación:

Importancia Relativa: expresa la importancia en tanto por ciento de una especie respecto a la densidad total de la comunidad (ALEGRE et AL., 1987). Se han reconocido las siguientes clases de importancia relativa: dominantes (ED), aquellas especies cuya densidad supone al menos el 5\% de la densidad global de la comunidad; influyentes (EI), cuya densidad supone entre un 5 y 
un $2 \%$ de la densidad global; recesivas (ER), cuya densidad es inferior al $2 \%$ de la densidad global.

Tipo de Distribución: indica, en tanto por ciento, la variación mensual de la distribución de las especies en la comunidad, en función su presencia en cada una de las estaciones de muestreo (VELASCO y BLANCO, 1996). Se reconocen los siguientes tipos de distribución: Amplia distribución (especies presentes en más de $75 \%$ de las muestras), Media distribución (especies presentes en el 75-25\% de las muestras), Baja distribución (especies presentes en menos del $25 \%$ de las muestras).

Constancia: utilizado en diversos trabajos (ALEGRE et AL.; 1987; VELASCO y BLANCO, 1996), expresa en tanto por ciento el grado de fidelidad o constancia de las especies a lo largo del año en cada ribera. Se reconocen los siguientes umbrales: constantes (especies presentes en el 70-100\% de los meses que forman el periodo de análisis), irregulares (presentes en el 69$20 \%$ de los meses que forman el periodo de análisis), esporádicas (presentes en menos del $20 \%$ de los meses que forman el periodo de análisis).

\section{RESULTADOS}

\section{III.1. Riqueza y densidad}

La densidad de la comunidad de aves acuáticas del río Jarama, expresada como IKA (Índice Kilométrico de Abundancia: $\mathrm{n}^{\circ}$ de aves $/ \mathrm{km}$ de río) experimenta una acusada variación anual (CUADRO 3, FIGURA 1), como así lo demuestra la amplia diferencia existente entre los valores extremos de este parámetro, con IKA máximos registrados en los meses invernales (diciembre: 192,72 aves $/ \mathrm{km}$, enero: 293,28 aves $/ \mathrm{km})$ y en el mes de julio $(228,32$ aves $/ \mathrm{km})$ e IKA mínimos marcados en los meses de marzo (80,72 aves $/ \mathrm{km})$ y mayo $(78,48$ aves $/ \mathrm{km})$, siendo el valor medio de este parámetro de 155,77 aves $/ \mathrm{km}( \pm 63,46)$. Los valores más elevados de riqueza máxima $\left(\mathrm{r}_{\mathrm{M}}\right)$, riqueza mínima $\left(\mathrm{r}_{\mathrm{m}}\right)$ y riqueza total $\left(\mathrm{r}_{\mathrm{t}}\right)$ se registraron, respectivamente, en los meses de febrero (22), agosto (10) y agosto (31); mientras que el valor de riqueza media (r) más elevado se alcanzó en los meses de julio, agosto y septiembre $(13,00)$ (CUADRO 3). La riqueza total $(x=25,00 \pm 3,37 ; n=12)$, cuyo valor anual acumulado es de 54, registra valores máximos en los meses de febrero y agosto (31) y valores mínimos en enero-diciembre (21) y junio (23), en el resto de los meses las variaciones en el valor de este parámetro son inferiores a 2 puntos. Se observa por tanto que no existe una relación directa entre los meses en los que el valor de la densidad es más elevado (invierno) y los meses de mayor riqueza (pasos migratorios). 
La explicación de los elevados valores de densidad en los meses invernales, que triplican a los que se registran durante el periodo de reproducción, se deben a la presencia en esta ribera de un nutrido grupo de especies invernantes, que en algunos casos se suman a las poblaciones locales, pero que en otros son elementos exclusivamente invernantes. Los elevados valores del mes de julio están relacionados con la presencia de individuos inmaduros pertenecientes a las tres especies más frecuentes de esta ribera: Anas platyrhynchos, Fulica atra y Gallinula chloropus. Por el contrario, los meses en los que los valores de riqueza son más elevados coinciden con los pasos migratorios, principalmente con el postnupcial, que se produce en agostoseptiembre.

En el río Tajo, los valores de densidad más elevados se registran durante el invierno, en los meses de noviembre $(198,57 \mathrm{aves} / \mathrm{km})$ y diciembre $(203,05$ aves $/ \mathrm{km}$ ), mientras que los más bajos coinciden con el periodo de reproducción, en mayo $(65,02$ aves $/ \mathrm{km})$ y junio $(80,79$ aves $/ \mathrm{km})$, siendo el valor medio anual del IKA 139,29 aves $/ \mathrm{km}$. Los valores de riqueza son más heterogéneos que los de densidad: la riqueza mínima más elevada se alcanza en enero (9) y febrero (7), y la riqueza máxima en febrero y mayo (22), aunque se encuentran muy cerca los meses de junio, agosto y septiembre (21) y noviembre-diciembre (22). Los valores de riqueza media varían poco, aunque los máximos se registran en diciembre y enero $(10,15)$, mientras que los valores de riqueza total más altos corresponden a los meses de abril y enero (28), y agosto y noviembre (25).

Los valores de riqueza total, cuyo valor anual acumulado es de 43 , son bastante homogéneos $(x=22,83 \pm 2,85 ; n=12)$, con máximos alcanzados en el mes de abril (25) y mínimos registrados en el mes de junio (18). En esta ribera se aprecia, como en la del Jarama, que las máximas concentraciones de aves acuáticas se producen durante los meses invernales, mientras que los valores mínimos de densidad corresponden a la estación reproductora.

La distribución anual de la riqueza está más repartida y no muestra variaciones tan claras como la densidad: las variaciones de riqueza media son poco acusadas, aunque los valores más altos corresponden siempre a los meses invernales, mientras que la riqueza total más alta se produce en el paso migratorio postnupcial, igual que la riqueza máxima. Como en el Jarama, no existe una relación directa entre los meses de alta densidad y los meses de elevada riqueza, si bien, los meses de baja riqueza se relacionan positivamente con los de baja densidad.

La comunidad de aves acuáticas del río Ebro representa un caso particular respecto a los anteriores. Los valores de densidad son claramente más bajos 
que en los ríos Tajo y Jarama, los máximos se producen en los mes de septiembre $(35,05$ aves $/ \mathrm{km})$ y octubre $(39,17$ aves $/ \mathrm{km})$, mientras que los mínimos corresponden a los meses de enero (6,03 aves/km) y junio $(7,14$ aves $/ \mathrm{km})$. El valor medio del IKA es también muy bajo en comparación con los registrados en el Tajo y en el Ebro (19,73 aves/km, $\pm 10,29)$. Los valores de riqueza más elevados coinciden, como los de densidad, con los pasos migratorios: riqueza máxima en abril (13), riqueza mínima en agostoseptiembre (4), riqueza media en septiembre $(8,00)$ y riqueza total en abril (27) y septiembre (25). La riqueza total $(x=17,83 \pm 4,60 ; n=12)$, cuyo valor anual acumulado es de 39, experimenta variaciones más acusadas que en los dos casos anteriores; si bien, sus máximos coinciden claramente con los pasos migratorios y suceden en los meses de abril (27) y septiembre (25), mientras que los mínimos se producen durante la invernada, en el mes de enero (13), y en el periodo de reproducción, en los meses de mayo (13) junio (11).

La invernada de aves acuáticas en el río Ebro es, por tanto, poco importante, siendo los pasos migratorios, especialmente el postnupcial, el periodo en que las concentraciones de aves son mayores.

Cuadro 3. Variación anual de la riqueza y densidad de las comunidades de aves acuáticas de los ríos, Ebro y Tajo-Jarama.

\begin{tabular}{|c|c|c|c|c|c|c|c|c|c|c|c|c|c|}
\hline & & $E$ & $F$ & $M$ & $A$ & $M$ & $J$ & $J l$ & $A$ & $S$ & $O$ & $N$ & $D$ \\
\hline \multirow[t]{4}{*}{$\mathrm{L}$} & $\mathrm{T}$ & 49,9 & 19,3 & 20,3 & 20,3 & 20,3 & 20,3 & 20,3 & 20,3 & 20,3 & 20,3 & 20,3 & 20,3 \\
\hline & $\mathrm{J}$ & 12,5 & 12,5 & 12,5 & 12,5 & 12,5 & 12,5 & 12,5 & 12,5 & 12,5 & 12,5 & 12,5 & 12,5 \\
\hline & $E$ & 22,4 & 23,2 & 22,4 & 23,2 & 22,4 & 22,4 & 16,0 & 22,4 & 20,2 & 24,6 & 23,2 & 23,2 \\
\hline & $\mathrm{T}$ & 32 & 12 & 13 & 13 & 13 & 13 & 13 & 13 & 13 & 13 & 13 & 13 \\
\hline \multirow[t]{3}{*}{$n$} & $\mathrm{~J}$ & 6 & 6 & 6 & 6 & 6 & 6 & 6 & 6 & 6 & 6 & 6 & 6 \\
\hline & $E$ & 10 & 11 & 10 & 11 & 10 & 10 & 8 & 10 & 9 & 12 & 11 & 11 \\
\hline & $\mathrm{T}$ & 14 & 13 & 13 & 13 & 11 & 12 & 16 & 16 & 11 & 14 & 16 & 16 \\
\hline \multirow[t]{3}{*}{$r_{M}$} & $\mathrm{~J}$ & 15 & 22 & 14 & 13 & 22 & 13 & 21 & 16 & 21 & 21 & 20 & 20 \\
\hline & $\mathrm{E}$ & 8 & 8 & 9 & 13 & 7 & 8 & 14 & 10 & 12 & 10 & 9 & 9 \\
\hline & $\mathrm{T}$ & 9 & 7 & 6 & 6 & 6 & 6 & 4 & 4 & 4 & 5 & 6 & 4 \\
\hline \multirow[t]{3}{*}{$r_{m}$} & $\mathrm{~J}$ & 6 & 5 & 7 & 7 & 6 & 6 & 8 & 10 & 6 & 4 & 7 & 7 \\
\hline & $E$ & 0 & 1 & 1 & 2 & 3 & 0 & 1 & 4 & 4 & 4 & 3 & 2 \\
\hline & $\mathrm{T}$ & 9,59 & 9,08 & 8,92 & 8,77 & 7,85 & 8,00 & 9,85 & 9,85 & 8,00 & 8,92 & 9,46 & 10,15 \\
\hline \multirow[t]{3}{*}{$r$} & $\mathrm{~J}$ & 10,67 & 10,33 & 10,00 & 11,00 & 10,00 & 10,33 & 13,00 & 13,00 & 13,00 & 12,50 & 11,17 & 11,07 \\
\hline & $E$ & 2,20 & 4,27 & 5,80 & 7,18 & 4,50 & 3,50 & 6,00 & 7,30 & 8,00 & 6,67 & 6,09 & 4,91 \\
\hline & $\mathrm{T}$ & 25 & 24 & 20 & 28 & 20 & 18 & 23 & 25 & 19 & 24 & 25 & 23 \\
\hline \multirow[t]{3}{*}{$r_{t}$} & $\mathrm{~J}$ & 21 & 31 & 24 & 25 & 24 & 23 & 25 & 31 & 29 & 24 & 22 & 21 \\
\hline & $E$ & 13 & 15 & 19 & 27 & 13 & 11 & 19 & 20 & 25 & 9 & 15 & 18 \\
\hline & $\mathrm{T}$ & 152,27 & 156,94 & 108,82 & 103,45 & 65,02 & 80,79 & 137,19 & 164,33 & 107,54 & 193,50 & 198,57 & 203,05 \\
\hline \multirow[t]{2}{*}{$I K A$} & $\mathrm{~J}$ & 293,28 & 170,00 & 80,72 & 93,28 & 78,48 & 88,40 & 228,32 & 184,48 & 122,32 & 180,08 & 157,12 & 192,72 \\
\hline & $\mathrm{E}$ & 6,03 & 10,04 & 15,80 & 16,94 & 10,00 & 7,14 & 20,38 & 24,64 & 35,05 & 39,17 & 25,00 & 26,59 \\
\hline
\end{tabular}

L: longitud de los tramos de censo en km. $\mathrm{n}$ : Número de tramos censados. $\mathrm{r}_{\mathrm{M}}$ : riqueza máxima; riqueza de la estación de riqueza más elevada. $\mathrm{r}_{\mathrm{t}}$ : riqueza total; número total de especies. $\mathrm{r}_{\mathrm{m}}$ : riqueza mínima; riqueza de la estación de riqueza más baja. $\mathrm{r}$ : Riqueza media; número medio de especies por estación. IKA: Índice Kilométrico de Abundancia (aves/km).

T, Tajo; J, Jarama; E, Ebro. 
Figura 1. Evolución mensual de la densidad (aves $/ \mathrm{km}-I K A-)$

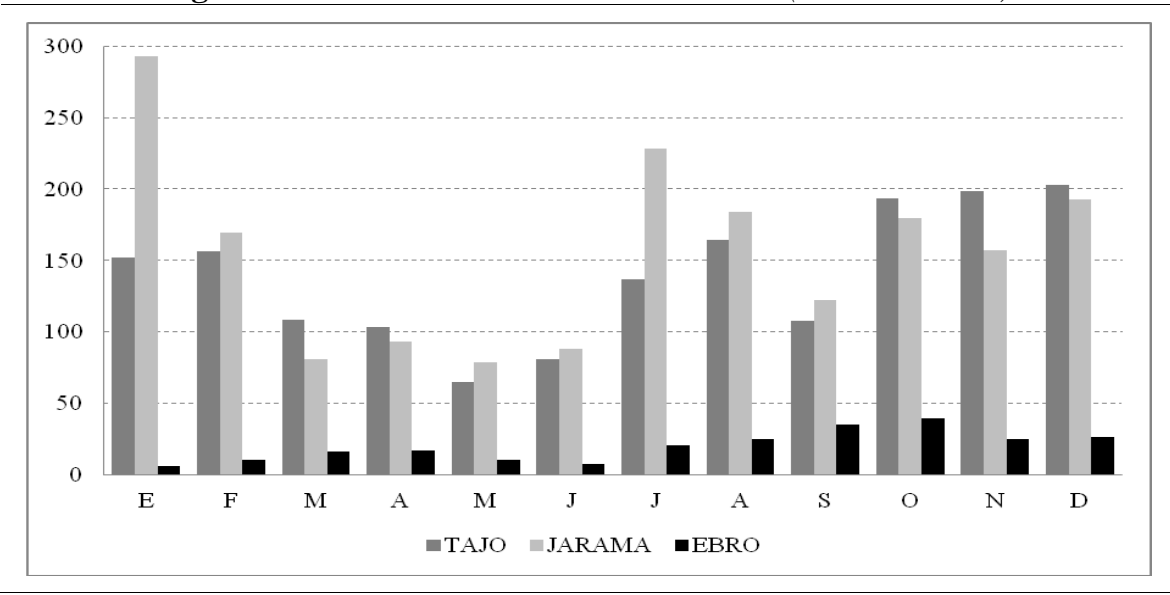

\section{III.2. Constancia y fenología}

La estructura de las comunidades de aves acuáticas, en función del grado de fidelidad de sus elementos a lo largo del año, presenta una distribución relativamente homogénea, como puede observarse en el Cuadro 4. En los tres casos son las especies irregulares los elementos más numerosos, aunque el segundo lugar en importancia lo ocupan las especies constantes en el Tajo y en el Jarama, y las especies esporádicas en el Ebro.

En el Ebro son elementos constantes las siguientes especies: Phalacrocorax carbo, Ardea cinerea, Ciconia ciconia, Anas platyrhynchos, Circus aeroginosus, Gallinula chloropus, Fulica atra, Charadrius dubius, Gallinago gallinago, Tringa ochropus y Alcedo atthis. En el Tajo y en el Jarama son especies constantes, comunes a ambas zonas, Tachybaptus ruficollis, Egretta garzeta, Ardea cinerea, Anas strepera, Anas platyrhynchos, Anas clypeata, Aythya ferina, Gallinula chloropus, Fulica atra, Himantopus himantopus, Actitis hypoleucos y Larus ridibundus, siendo Nyctycorax nyctycorax, Aythya fuligula y Vanellus vanellus especies constantes sólo en el río Tajo.

Cuadro 4. Constancia de las comunidades de aves acuáticas. Especies

\begin{tabular}{lrrrrrr}
\hline & Ebro & \multicolumn{3}{c}{ Tajo } & \multicolumn{2}{c}{ Jarama } \\
\hline & $n^{\circ}$ especies & $\%$ & $n^{o}$ especies & $\%$ & $n^{\circ}$ especies & $\%$ \\
\hline Constantes & 12 & 30,77 & 14 & 33,33 & 16 & 30,19 \\
Irregulares & 14 & 35,90 & 16 & 38,10 & 22 & 41,51 \\
Esporádicas & 13 & 33,33 & 12 & 28,57 & 15 & 28,30 \\
\hline Total & 39 & 100 & 42 & 100 & 53 & 100 \\
\hline
\end{tabular}

La estructura fenológica de las comunidades de aves acuáticas muestra mayores diferencias que las observadas en relación con la constancia 
(CUADRO 5). En el Ebro, las especies invernantes son las más numerosas $(30,77 \%)$, seguidas de las especies migrantes $(23,08 \%)$ y de las especies residentes $(23,08 \%)$. En el caso de esta ribera, es llamativo que el periodo fenológicamente más representativo (invierno) no coincida con el periodo en el que las densidades de aves acuáticas son más elevadas (pasos migratorios), aunque son varias las especies consideradas como invernantes cuya densidad es más elevada durante los pasos migratorios: Actitis hypoleucos, con densidades durante el mes de julio $(3,60 \mathrm{aves} / \mathrm{km})$ claramente más alta que las registradas en los meses de enero $(0,04$ aves $/ \mathrm{km})$ o febrero $(0,20$ aves $/ \mathrm{km})$, o las de Tringa ochropus, alta en septiembre (3,80 aves $/ \mathrm{km})$ y baja en los meses de enero y febrero $(0,10$ aves $/ \mathrm{km})$.

En el Tajo, los elementos residentes son los más numerosos $(38,10 \%)$, seguidos por las especies invernantes $(26,19 \%)$ y por las especies migrantes $(23,81 \%)$. Al igual que en el Ebro, muchas especies residentes aumentan de forma importante su densidad durante los meses invernales, como Anas platyrhynchos, cuya densidad durante el mes de noviembre es de 99,0 aves $/ \mathrm{km}$, siendo en el mes de junio de 21,8 aves $/ \mathrm{km}$; Fulica atra, alcanza valores máximos en noviembre-diciembre $(49,7 \mathrm{aves} / \mathrm{km})$ y mínimos en mayo $(14,0$ aves $/ \mathrm{km})$; o Aythya ferina, que registra una elevada densidad en febrero $(11,0 \mathrm{aves} / \mathrm{km})$, muy baja en septiembre $(0,10 \mathrm{aves} / \mathrm{km})$, octubre $(0,30$ aves $/ \mathrm{km})$ y abril (1,60 aves $/ \mathrm{km})$.

Cuadro 5. Fenología de las comunidades de aves acuáticas. Especies

\begin{tabular}{lrrrrrr}
\hline & Ebro & \multicolumn{3}{c}{ Tajo } & \multicolumn{3}{c}{ Jarama } \\
\hline & $n^{o}$ especies & $\%$ & $n^{\circ}$ especies & $\%$ & $n^{o}$ especies & $\%$ \\
\hline Residentes & 9 & 23,08 & 16 & 38,10 & 14 & 26,42 \\
Invernantes & 12 & 30,77 & 11 & 26,19 & 12 & 22,64 \\
Estivales & 4 & 10,26 & 5 & 11,90 & 6 & 11,32 \\
Migrantes & 10 & 25,64 & 10 & 23,81 & 19 & 35,85 \\
Ocasionales & 3 & 7,69 & - & - & 1 & 1,89 \\
Accidentales & 1 & 2,56 & - & - & 1 & 1,89 \\
\hline Total & 39 & 100 & 42 & 100 & 53 & 100 \\
\hline
\end{tabular}

En la comunidad de aves acuáticas del río Jarama las especies más frecuentes son elementos migrantes $(35,85 \%)$, seguidas por las especies residentes $(26,41 \%)$ y por las especies invernantes $(22,64 \%)$. La dominancia de las especies migrantes en esta comunidad se debe a la elevada importancia de este curso para un buen número de especies que sólo aparecen durante los pasos migratorios, aunque la importancia de estos elementos en términos de densidad es poco importante. Son, en concreto, varias especies de limícolas que sólo aparecen en los pasos migratorios (Charadrius alexandrinus, Charadrius hiaticula, Pluvialis squatarola, Calidris alpina, Calidris minuta, Ca- 
lidris alba, Tringa erythropus, Tringa glareola) u otras aves acuáticas (Anas querquedula, Gelochelidon nilotica o Chlidonias niger).

\section{III.3. Dominancia e importancia relativa}

La distribución mensual de la dominancia sigue en las tres riberas pautas muy parecidas: las especies recesivas son siempre los elementos más frecuentes, seguidas por las especies dominantes y por las especies irregulares.

En el Ebro, los valores de densidad de las especies dominantes oscilan entre un máximo del $91,43 \%(24,31$ aves $/ \mathrm{km})$, registrado en noviembre, y un valor mínimo de 71,44\% (14,56 aves $/ \mathrm{km})$ marcado en julio. El grupo de especies dominantes está integrado por 12 elementos, aunque sólo cuatro de ellos ostentan esta categoría de dominancia durante más de seis meses: Anas platyrhynchos (12 meses), Ardea cinerea (9 meses), Charadrius dubius (8 meses) y Phalacrocorax carbo (7 meses). Destacan por su elevada densidad los valores registrados por Phalacrocorax carbo en los meses de enero (3,04 aves $/ \mathrm{km} ; 58,21 \%)$ y febrero $(6,03$ aves $/ \mathrm{km} ; 60,0 \%)$, Charadrius dubius en abril $(6,68$ aves $/ \mathrm{km} ; 39,43 \%)$ y Anas platyrhynchos en agosto $(9,55$ aves/ $\mathrm{km} ; 38,76 \%)$.

En el Tajo, la densidad de las especies dominantes es máxima en marzo $(97,34$ aves $/ \mathrm{km} ; 89,45 \%)$ y septiembre $(97,14$ aves $/ \mathrm{km} ; 90,33 \%)$ y mínima en enero $(118,24$ aves $/ \mathrm{km} ; 79,80 \%)$ y febrero $(118,57$ aves $/ \mathrm{km} ; 77,87 \%)$. De las ocho especies que forman el grupo de especies dominantes, tres (Anas platyrhynchos, Fulica atra y Gallinula chloropus) son dominantes durante 12 meses, siendo las restantes dominantes durante 3 o menos meses. Destacan por su elevada importancia relativa Anas platyrhynhos en mayo (31,72 aves $/ \mathrm{km} ; 48,78 \%)$, septiembre $(43,94$ aves $/ \mathrm{km} ; 40,86 \%)$ y noviembre $(98,97$ aves $/ \mathrm{km} ; 49,84 \%)$, Fulica atra en junio $(25,81$ aves $/ \mathrm{km} ; 31,95 \%)$ y septiembre $(37,83$ aves $/ \mathrm{km} ; 35,18 \%)$ y Gallinula chloropus en abril $(26,35$ aves $/ \mathrm{km}$; $20,20 \%)$, agosto $(65,76$ aves $/ \mathrm{km} ; 40,01 \%)$ y enero $(26,87$ aves $/ \mathrm{km} ; 17,93$ $\%)$.

Los valores del Índice de Dominacia de Mcnaugthon y Wolf, una medida que debe ser entendida en sentido inverso a la Diversidad, siguen en las tres riberas pautas diferentes. En el Ebro el par de valores máximos se alcanza durante los meses de febrero $(71,12)$ y enero $(67,41)$ y el par de valores mínimos se registra en julio $(44,48)$ y septiembre $(43,26)$. En el Tajo, el par de valores máximos se produce en los meses de septiembre $(76,04)$ y noviembre $(74,89)$ y el par de valores mínimos en los meses de febrero $(48,69)$ y julio $(49,16)$. En el Jarama los valores máximos de este indicador suceden en 
enero $(70,97)$ y julio $(69,55)$ y los mínimos en agosto $(48,31)$ y noviembre $(49,08)$.

Cuadro 6. $N^{o}$ de meses en los que una especie es elemento dominante

\begin{tabular}{lccc}
\hline \multicolumn{1}{c}{ Especies } & Ebro & Tajo & Jarama \\
\hline Tachybaptus ruficollis & - & - & 4 \\
Phalacrocorax carbo & 7 & 3 & - \\
Ardea cinerea & 9 & - & - \\
Ardea purpurea & 1 & - & - \\
Bubulcus ibis & - & 1 & 1 \\
Ciconia ciconia & 1 & - & - \\
Anas crecca & 1 & - & 2 \\
Anas platyrhynchos & 12 & 12 & 9 \\
Anas clypeata & - & 2 & 1 \\
Anas strepera & - & - & 1 \\
Aythya ferina & - & 2 & 1 \\
Fulica atra & 2 & 12 & 12 \\
Gallinula chloropus & - & 12 & 12 \\
Vanellus vanellus & 1 & - & 2 \\
Himantopus himantopus & - & 3 & 4 \\
Charadrius dubius & 8 & - & 1 \\
Tringa ochropus & 5 & - & - \\
Actitis hypoleucos & 5 & - & 1 \\
Gallinago gallinago & 3 & - & - \\
Larus ridibundus & - & - & 5 \\
\hline
\end{tabular}

\section{III.4. Diversidad y equitabilidad}

La diversidad $(\mathrm{H})$ de la comunidad de aves acuáticas de la ribera del Ebro, basada en el índice de Shannon, alcanza valores máximos en los meses de julio $(\mathrm{H}=2,236)$ y septiembre $(\mathrm{H}=2,225)$, y mínimos en enero $(1,617)$ y febrero $(1,517)$, siendo el valor medio de este parámetro de 1,939 $( \pm 0,244$ $n=12)$. En el Tajo $(x=1,774 \pm 0,200 n=12)$, los valores de diversidad más elevados se registran en los meses de febrero $(\mathrm{H}=2,152)$ y diciembre $(H=1,960)$, mientras que los mínimos tienen lugar en septiembre $(H=1,440)$ y en mayo $(\mathrm{H}=1,589)$.

En el Jarama $(x=1,982 \pm 0,202, n=12)$, los valores más elevados se producen en agosto $(\mathrm{H}=2,259)$ y noviembre $(\mathrm{H}=2,121)$ y los mínimos en enero $(\mathrm{H}=1,632)$ y julio $(\mathrm{H}=1,573)$. Como es lógico, los valores de diversidad se relacionan negativamente con los del índice de dominancia, tanto en el Ebro $(r=-0,976 p<0,05 ; n=12)$, como en el Tajo $(r=-0914 ; p<0,05 n=12)$ y en el Jarama $(r=-0,968 ; p<0,05 ; n=12)$, aunque en el Jarama los meses de diversidad mínima no coinciden plenamente con los meses en los que el valor del índice de dominancia es más elevado. 
La equitabilidad (J), basada en Pielou, en la ribera del Ebro $(x=0,681 \pm 0,061 n=12)$, alcanza valores máximos en los meses de junio $(\mathrm{J}=0,786)$ y julio $(\mathrm{J}=0,759)$ y mínimos en los meses de febrero $(0,560)$ y mayo $(0,633)$. En el Tajo $(x=0,567 \pm 0,065, n=12)$, los valores máximos se registran en febrero $(\mathrm{J}=0,677)$ y diciembre $(\mathrm{J}=0,625)$ y los mínimos en abril $(\mathrm{J}=0,495)$ y noviembre $(\mathrm{J}=0,487)$. Por último, en el Jarama $(x=0,613 \pm 0,056$ $n=12)$, son máximos los valores de julio $(\mathrm{J}=0,660)$ y diciembre $(\mathrm{J}=0,676)$, y mínimos los de enero $(\mathrm{J}=0,536)$ y julio $(\mathrm{J}=0,489)$. Los valores de diversidad y equitabilidad se correlacionan positivamente en las tres zonas de estudio (Ebro: $\quad r_{s}=0,59 \quad p=0,049 ; \quad$ Tajo: $\quad r_{s}=0,95 \quad p=0,002 ; \quad$ Jarama: $r_{s}=0,83$ $p=0,006$ ), aunque los valores máximos de ambos parámetros no coinciden en todos los casos.

\section{III.5. Estructura trófica}

El grupo trófico más importante en la comunidad de aves acuáticas de la ribera del Ebro está formado por especies consumidoras de invertebrados, dominantes durante siete meses, seguido de las especies ictiófagas, dominantes durante tres meses y, por último por las especies consumidoras de materia vegetal e invertebrados que son dominantes únicamente en un mes (agosto). La importancia relativa de las especies consumidoras de invertebrados en los meses en que son la categoría trófica dominante, calculada en porcentaje sobre la densidad total mensual (aves/ $\mathrm{km}$ ), oscila entre un máximo absoluto del 64,67\% registrado en el mes septiembre y un máximo mínimo (máximo más bajo) del 50,35\% en abril. La importancia de las especies ictiófagas varía entre un máximo absoluto del $71,71 \%$, alcanzado en febrero, y un máximo mínimo del 37,95\%, registrado en marzo. La importancia relativa de las especies consumidoras de materia vegetal e invertebrados en el único mes que son la categoría dominante es del $40,95 \%$.

La estructura trófica de las comunidades de aves acuáticas de los ríos Tajo y Jarama es más simple que la del Ebro. Las especies consumidoras de materia vegetal e invertebrados son dominantes en el Tajo durante todo el periodo de análisis, mientras que en el Jarama, esta categoría trófica es dominante en once meses, y sólo en uno son dominantes las especies consumidoras de invertebrados. Los valores extremos máximos de la densidad de las especies consumidoras de materia vegetal e invertebrados en el Tajo oscilan entre un máximo del 90,38\% registrado en septiembre y un mínimo del 61,10\% marcado en diciembre. En el Jarama, los valores máximos de esta categoría oscilan entre un máximo absoluto del 85,63\% del mes de marzo y un máximo mínimo del 46,68\% registrado en el mes de febrero; la importancia relativa de las especies consumidoras de invertebrados es del 50,41\% en el único mes en que esta categoría es dominante. 
Cuadro 7. $N^{o}$ de meses en los que una categoría trófica es dominante

\begin{tabular}{lccc}
\hline & Ebro & Tajo & Jarama \\
\hline Ictiófagos & 4 & - & - \\
Mat.Vegetal-Invertebrados. & 1 & 12 & 11 \\
Invertebrados & 7 & - & 1 \\
\hline
\end{tabular}

\section{III.6. Distribución}

Como puede observarse en el CUADRO 8, las especies de baja distribución son dominantes durante 11 meses en la ribera del Ebro; sólo en un mes (agosto), las especies de media distribución se sitúan como clase dominante. En las riberas del Tajo y del Jarama, esta categoría (baja distribución) aparece como dominante, respectivamente, durante 8 y 6 meses, mientras que las especies de media distribución dominan durante 4 meses en el Tajo y durante 6 meses en el Jarama. En el Ebro, las especies de baja distribución representan siempre más del $40 \%$ de la riqueza total mensual, con máximos en enero $(84,62 \%)$ y mínimos en agosto $(40 \%)$. Tanto en el Tajo como en el Jarama, la distribución por categorías está más repartida: valores de riqueza máxima en $\%$ de esta clase representan el $50 \%$ en el Tajo (mayo) y en el Jarama (marzo).

Cuadro 8. $N^{\circ}$ de meses en los que una categoría de distribución es dominante

\begin{tabular}{lccc}
\hline & Ebro & Tajo & Jarama \\
\hline Amplia Distribución & - & - & - \\
Media Distribución & - & 4 & 6 \\
Baja Distribución & 12 & 8 & 6 \\
\hline
\end{tabular}

Cuadro 9. $N^{o}$ de meses en los que una especie es elemento de amplia distribución

\begin{tabular}{lccc}
\hline \multicolumn{1}{c}{ Especies } & Ebro & Tajo & Jarama \\
\hline Tachybaptus ruficollis & - & 11 & 9 \\
Phalacrocorax carbo & - & 4 & - \\
Ardea purpurea & 1 & - & - \\
Ardea cinerea & 6 & 5 & - \\
Anas crecca & - & - & 1 \\
Anas platyrhynchos & 2 & 12 & 10 \\
Anas clypeata & 1 & 3 & 1 \\
Gallinula chloropus & - & 12 & 12 \\
Fulica atra & - & 12 & 12 \\
Tringa ochropus & 5 & - & 5 \\
Gallinago gallinago & - & - & 1 \\
Himantopus himantopus & - & 3 & 5 \\
Charadrius dubius & 2 & 3 & 2 \\
Actitis hypoleucos & 2 & 2 & 2 \\
\hline
\end{tabular}




\section{DISCUSIÓN}

\section{IV.1. Riqueza, densidad, diversidad y equitabilidad}

Los valores de densidad mensual de las comunidad de aves acuáticas del Tajo y del Jarama no muestran diferencias apreciables a lo largo del ciclo anual $(z=0,823 ; p=0,410)$; sin embargo, si existen diferencias significativas entre el Ebro y el Tajo $(z=3,099 ; p=0,0019)$, y entre el Ebro y el Jarama $(z=3,099 ; p=0,0019)$. Las diferencias que se producen en los valores de densidad de las comunidades de aves acuáticas de estas tres riberas son especialmente llamativas, no sólo por el desigual valor de este parámetro, sino también por la desigual distribución de los máximos en cada ribera: los valores máximos del Tajo $(203,05$ aves $/ \mathrm{km})$ y del Jarama $(293,28 \mathrm{aves} / \mathrm{km})$, contrastan fuertemente con los que se producen en el Ebro $(39,17$ aves $/ \mathrm{km})$. Además, las densidades más elevadas en el Tajo y en el Jarama se registran durante los meses invernales, mientras que los máximos del Ebro coinciden con los meses en los que se producen los pasos migratorios.

Se considera que las grandes diferencias existentes en los valores de densidad están relacionadas con dos factores ambientales: las características hidrológicas e hidráulicas y la calidad del agua. El río Ebro es un curso poco regulado por grandes embalses, característica que provoca importantes crecidas anuales, cuyas puntas de caudal, superiores con frecuencia a los 2.000 $\mathrm{m}^{3} / \mathrm{s}$, se registran siempre en los meses de diciembre y enero, y más raramente en abril y mayo. La intensidad de las avenidas de recurrencia anual en el sistema Tajo-Jarama es claramente más baja que en el curso anterior debido a la elevada capacidad de embalse de la cuenca en los tramos que afectan al área de estudio. En este sector de la cuenca hidrográfica el volumen regulado supera en más de un $20 \%$ a la aportación media anual del río Tajo en la ciudad de Toledo, el tramo fluvial del área de estudio donde este curso alcanza su mayor caudal (MOLINA y BERROCAL, 2006). Por otra parte, en el río Ebro dominan los tramos de régimen rápido, mientras que en el Tajo y en el Jarama son dominantes los tramos de régimen lento, lo que ha favorecido el desarrollo de extensos cinturones de vegetación palustre en la orilla aluvial, integrados principalmente por Phragmites australis y Typha domingensis, hábitats de gran interés para aves acuáticas, más escasos sin embargo en el Ebro.

El desigual comportamiento hidrológico e hidráulico de los tres ríos analizados determina diferentes potencialidades para las comunidades de aves acuáticas. Es preciso considerar además otro importante factor ambiental para explicar las grandes diferencias que se producen en los valores de densidad: la calidad de las aguas. La elevada carga de nutrientes de los ríos Tajo y 
Jarama, claramente más elevada que la del Ebro, favorece el desarrollo de varias especies de algas, plantas vasculares e invertebrados acuáticos que forman parte de la dieta de las especies más abundantes en el Tajo-Jarama: Anas platyrhynchos, Fulica atra y Gallinula chloropus, especies cuya densidad supone más del $50 \%$ de la densidad total durante los meses invernales en los ríos Tajo y Jarama, pero menos del $23 \%$ en el Ebro.

Los valores de riqueza muestran en general importantes diferencias, salvo los valores de riqueza mínima y riqueza total del Tajo y del Jarama. Los valores más altos de $r_{t}$ se registran en los tres casos durante los pasos migratorios, debido al tránsito de un buen número de aves limícolas, igual que sucede en otros ríos peninsulares (HERNÁNDEZ y VELASCO, 1990). En general, los valores más altos de riqueza se alcanzan siempre en el río Jarama, seguido por el río Tajo y, en último lugar, por el río Ebro. Este hecho parece estar en relación con las características de la orilla aluvial, siempre más compleja y heterogénea en el río Jarama que en los restantes cursos.

En este caso complejidad no es sinónimo de estado de conservación favorable, ya que la orilla aluvial del Jarama es con diferencia la más deteriorada de las tres riberas estudiadas. La orilla aluvial y el cauce del río Ebro, a pesar de su buen estado de conservación, son medios estructuralmente poco complejos: en el cauce de este curso dominan los tramos de régimen rápido, en la orilla las superficies de gravas o arenas desnudas ocupan gran extensión y son muy escasas las áreas con vegetación palustre en las márgenes. La orilla aluvial y el cauce del río Jarama, por el contrario, es un complejo mosaico donde alternan los tramos de régimen rápido y lento, a diferencia de lo que sucede en las orillas del Tajo existen extensos depósitos de meandro y fangares carentes de vegetación, así como formaciones de vegetación palustre en las márgenes de gran densidad y longitud. El río Tajo representa un caso intermedio entre los dos anteriores, y aunque las características de su orilla aluvial se aproximan más a las del Jarama, no cuenta con superficies extensas de gravas desnudas y fangares, el hábitat más utilizado por un buen número de limícolas durante los pasos migratorios y durante el periodo postreproductor, como ya ha indicado VELASCO (1992).

Cabe señalar además, la importancia de los ríos Tajo y Jarama durante los ciclos secos. Los valores globales de anátidas y fochas registrados durante el invierno de 1995, año que marca el final de un ciclo particularmente seco en la Meseta meridional (GALÁN, 2004), no han sido superados hasta la fecha, como puede observarse en el gráfico posterior. En él se muestran diversos censos equiparables por su metodología y extensión censada a los realizados por los autores de este trabajo. 
Figura 2. Evolución de las cifras globales de anátidas y fochas invernantes en el río Tajo aguas abajo de Aranjuez (Madrid-Toledo) en el periodo 1995-2006

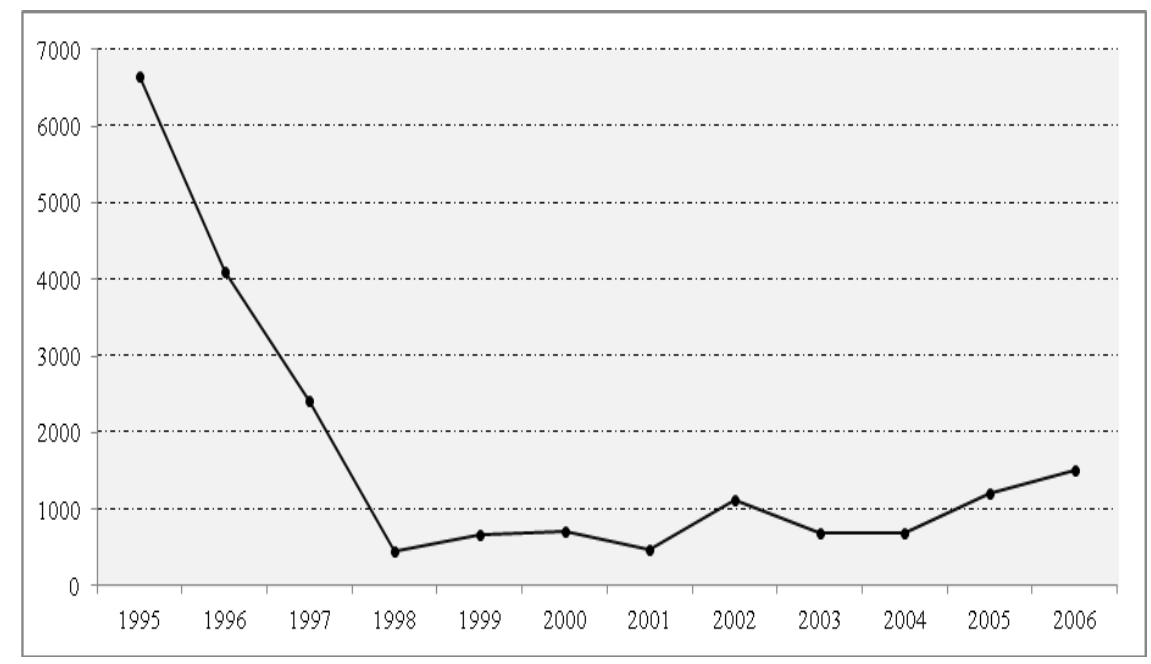

Fuente: Censo 1995, datos propios. Censos 1996-2006: SEO-BIRDLIFE, 1997-1999; DEL MORAL, 2000-2003; MOLINA, 2004-2007.

Los valores de diversidad $(\mathrm{H})$ de las comunidades de aves acuáticas no muestran diferencias apreciables entre el Tajo y el Ebro $(z=1,8439 p=0,066)$ y entre el Jarama y el Ebro $(z=0,8236 p=0,4101)$, aunque si entre el Tajo y el Jarama $(z=2,0003 p=0,0454)$, si bien estas diferencias no son especialmente significativas. Mayores son sin embargo las diferencias existentes en los valores mensuales de la equitabilidad (J), significativas entre el Ebro y el Jarama $(z=2,549 p=0,0107)$ y especialmente entre el Tajo y el Ebro $(z=2,706 p=0,0068)$, aunque no entre el Tajo y el Jarama $(z=1,451$ $p=0,146)$.

A priori, puede resultar llamativo que los valores medios anuales de diversidad y equitabilidad de la ribera del Ebro sean superiores a los del Tajo y del Jarama, a pesar de que los valores medios anuales y los totales mensuales de riqueza y densidad son mucho más elevados en estas dos riberas que en la del Ebro. En este caso, estas diferencias en los valores de $\mathrm{H}$ y J en las comunidades de las tres zonas de estudio pueden entenderse en función de la desigual distribución de la importancia relativa de las especies en la comunidad. La riqueza y densidad total mensual es siempre más elevada en el Tajo y en el Jarama que en el Ebro, pero en los dos primeros cursos, muy pocas especies, generalmente tres (Anas platyrhynchos, Gallinula chloropus, Fulica atra), alcanzan densidades muy elevadas, mientras que la distribución de la densidad específica en el Ebro suele estar más repartida. 
En definitiva, esta diferencia en los valores de estos parámetros es una manifestación del desigual estado de conservación de las tres zonas de estudio. Algunos importantes elementos del medio en el Tajo y en el Jarama, como por ejemplo la calidad de las aguas superficiales, muestran importantes síntomas de deterioro, que favorecen a unas pocas especies de la comunidad en detrimento de la mayoría de las restantes, hecho que se refleja en su elevada densidad. En el Ebro, la ausencia de un factor de alteración como el señalado provoca una distribución más equilibrada de la densidad específica que tiene su reflejo en unos valores más elevados de $\mathrm{H}$ y J.

\section{IV.2. Importancia relativa y estructura trófica}

La distribución de la importancia relativa de los elementos por categorías de dominancia y clases tróficas en la comunidad de aves acuáticas de estas riberas está también en relación con las características ecológicas de los tres ríos estudiados.

La distribución de la riqueza por categorías de dominancia no muestra diferencias apreciables entre las tres zonas, aunque sí que existen diferencias importantes, cuantitativas y cualitativas, en la composición de la categoría especies dominantes. En conjunto, la distribución trófica específica anual ( ${ }^{\circ}$ de especies por categoría trófica) no muestra diferencias importantes entre la comunidad de aves acuáticas del río Ebro y del río Tajo $\left(\chi 2_{2}=0,5222\right.$ $p=0,9975)$, ni entre la del Tajo y el Jarama $\left(\chi 2_{2}=1,8272 p=0,9349\right)$, ni tampoco entre los ríos Ebro y Jarama $\left(\chi_{2}=1,2136 p=0,9762\right)$; no obstante, las diferencias mensuales en función de la distribución de la densidad por categorías tróficas son mayores, especialmente entre las comunidades de aves acuáticas de los ríos Ebro y Jarama.

La distribución de la riqueza total mensual por grupos de categoría de dominancia en las tres riberas no muestra diferencias significativas, aunque la composición del grupo de especies dominantes por meses del año $\left(\mathrm{n}^{\circ}\right.$ de meses en los que una especie es elemento dominante) se correlaciona negativamente entre en el Tajo y el Ebro y entre el Jarama y el Ebro, aunque no entre el Tajo y el Jarama.

La elevada importancia relativa en el Tajo y en el Jarama de las especies incluidas en la categoría trófica materia vegetal-invertebrados, principalmente Anas platyrhynchos, Fulica atra y Gallinula chloropus, está relacionada con la alteración de la calidad de las aguas de estos cursos. Por este mismo motivo, la importancia relativa de las especies ictiófagas es en general baja o muy baja, aunque la mayor calidad de las aguas del Tajo respecto a las del Jarama se refleja en la presencia de Phalacrocorax carbo como espe- 
cie dominante durante 4 meses, especie ausente en el Jarama en esta categoría de dominancia.

En el Ebro, al contrario de lo que sucede en los ríos Tajo y Jarama, la dominancia de la categoría trófica ictiófagos está en relación con la mayor calidad de las aguas de este curso, que repercute positivamente en el tamaño de las poblaciones de peces que constituyen la base de la dieta de estos elementos. La abundancia de especies pertenecientes a la categoría invertebrados se relaciona con la importancia de este curso para algunas especies de aves limícolas durante el periodo de reproducción y los pasos migratorios (Charadrius dubius), durante la invernada y los pasos migratorios (Gallinago gallinago, Tringa ochropus, Actitis hypoleucos).

Phalacrocorax carbo, Ardea cinerea y Ardea purpurea, son especies dominantes durante 7, 9 y 1 meses respectivamente, mientras que Charadrius dubius, Tringa ochropus, Actitis hypoleucos y Gallinago gallinago son especies dominantes durante 8, 5, 5 y 3 meses respectivamente. Anas platyrhynchos, especie incluida en la categoría trófica materia vegetal-invertebrados, es especie dominante durante 12 meses. En este caso, este hecho puede estar en relación con la gran valencia ecológica de la especie (CRAMP, 1998; NUMMI et $A L$. 1994). Por su relevancia ecológica, es especialmente significativa la escasa importancia relativa de Fulica atra y Gallinula chloropus, precisamente las especies dominantes más comunes del Tajo y del Jarama.

Los hábitos tróficos de algunas de las especies dominantes expresan bien las relaciones existentes entre abundancia y calidad de las aguas. La dieta de Phalacrocorax carbo en el Sureste de Madrid, en el río Jarama y en las graveras de su llanura aluvial, es poco diversa y está compuesta fundamentalmente por Cyprinus carpio (64\%), Barbus sp. (16,5\%), Ictalurus melas (5\%) y ciprínidos indeterminados $(13,7 \%)$, siendo el tamaño medio de las presas de 132,5 $\pm 4,5 \mathrm{~mm}$ (BLANCO et AL., 1995). En otras zonas de la Península, como la ría y el estuario del Bidasoa (LEKUONA y CAMPOS, 1996) o en áreas más lejanas como el delta del Poo (BOLDREGHINI et AL., 1993), la dieta invernal de la especie está mucho más diversificada debido, entre otros aspectos, a la mayor diversidad y riqueza de las comunidades de peces de estos ámbitos.

En el valle del Jarama, las zonas de alimentación más importantes se sitúan en las graveras de la vega, mientras que en el Ebro y en el Tajo la especie ocupa toda la longitud de los cursos. La baja densidad de las poblaciones de peces del Jarama, un curso cuyo sector situado en el área de estudio fue considerado como sin vida piscícola estable (CUBILlO et AL., 1990) y que en la actualidad no ha mejorado sustancialmente, condiciona el tamaño de la po- 
blación invernante de esta especie. En el Tajo y en el Ebro debe de existir una correlación positiva entre la disponibilidad de recursos tróficos y el tamaño de la población invernante, como así lo demuestra la mayor densidad de esta especie en estas riberas, si bien el tamaño y la diversidad de las poblaciones de peces del río Tajo son bajos o muy bajos (CUBILlo et AL., 1990) en comparación con el río Ebro. La población de Phalacrocorax carbo, no obstante, ha experimentado un notable incremento desde la última década de 1990: en Madrid, se sitúo en 2003 en 1.740 individuos, 400 aves más que en 1998 (DE LA PUENTE y PONCE, 2004); en Toledo, donde el río Tajo concentra al 52\% de la población, ha pasado 2.120 individuos en 1996 a 4.220 en 2003 (MORENO-OPO, 2009). Apoya la hipótesis anterior otro argumento, relacionado con el proceso de colonización de Phalacrocorax carbo de la cuenca de los ríos Tajo y Jarama, tras la expansión de la especie desde los inicios de la década de los años 80, como consecuencia del aumento de la población reproductora del norte y centro de Europa (DEBOUT, 1987; BOLDREGHINI et AL., 1993; GREGERSEN, 1991; Røv, 1991; ZIJLSTRA y EERDEN, 1991; ZIMMERMAN y RUTSCHKE, 1991). La colonización de los ríos Tajo y Jarama se produjo con posterioridad a la ocupación de las graveras, los enclaves de mayor importancia trófica y además el hábitat acuático mejor conservado en el fondo de estos valles (BLANCO et AL., 1994).

En la ribera del Ebro, por el contrario, toda población de Phalacrocorax carbo se concentra en el río, ante la ausencia de otros hábitats adecuados en el fondo del valle. Aquí, el incremento del tamaño de la población invernante se ha realizado fundamentalmente a costa del aumento de la población que ocupaba las localidades tradicionales de invernada, lo que parece demostrar que el río Ebro aún mantiene recursos tróficos suficientes para mantener a una población de cormoranes en constante aumento. No obstante, algunos trabajos han señalado la ocupación de nuevos dormideros, aunque éstos se sitúan en hábitats de características similares a las de los hábitats tradicionalmente ocupados (CAMPOS y LEKUONA, 1994; LEKUONA y CAMPOS, 1996).

Algo similar sucede con otras especies ictiógafagas como Ardea cinerea, Ardea purpurea o Alcedo atthis, cuya importancia relativa es siempre mayor en el Ebro que en el Tajo y en el Jarama. El caso de Alcedo atthis es significativo debido a los grandes contrastes existentes en su densidad y constancia entre las tres zonas de estudio, principalmente entre el Ebro y el TajoJarama, considerando conjuntamente estos dos últimos cursos. Esta especie es un elemento constante en la ribera del Ebro, aunque sus máximas densidades se producen en los pasos migratorios, coincidiendo con el tránsito de aves invernantes procedentes en su mayor parte del centro de Europa 
(MARTín y PÉREZ, 1990). En el Tajo y en el Jarama Alcedo atthis es principalmente ave de paso, siendo muy escasas las citas plenamente invernales en los cursos, aunque es un elemento frecuente en las graveras de la vega durante este periodo del año, y más escasas aún la citas durante el periodo de reproducción. La mala calidad de las aguas de estos cursos, que repercute negativamente en la disponibilidad de recursos tróficos (BIRDLIFE INTERNATIONAL, 2004), se manifiesta en unas densidades muy bajas y en una distribución irregular de esta especie, cuya presencia se concentra en los pasos migratorio en el Tajo-Jarama. En el Ebro, las densidades de esta especie son siempre más elevadas y su distribución mensual es continua ya que, como ha señalado MEADOWs (1972) para los ríos de Gales e Inglaterra, existe una correlación directa entre altos niveles de contaminación fluvial (pérdida de recursos tróficos) y bajas densidades de la especie. En efecto, las poblaciones de peces del Tajo y del Jarama son pobres y poco diversas debido al alto grado de contaminación fluvial de estos cursos y, salvo Gambusia holdbrooki, son muy escasas o inexistentes las especies cuya talla se sitúa en el rango de tamaño $30-50 \mathrm{~mm}$, el característico de las presas principales de esta especie (CRAMP, 1998).

Ardea purpurea también es más frecuente en la ribera del Ebro que en el Tajo y en el Jarama, aunque esta especie sólo utiliza el río Ebro como lugar de alimentación. Las zonas de reproducción de esta ardeida se sitúan principalmente en zonas húmedas de la vega y en balsas y lagunas situadas en las vertientes próximas al fondo del valle, tanto en Navarra como en Aragón (BERGAANDI et AL., 1995; SEO-ARAGÓN, 1994, 1997).

La dieta de las tres especies de aves acuáticas más frecuentes en el TajoJarama, Gallinula chloropus, Fulica atra y Anas platyrhynchos, está constituida principalmente por materia vegetal y, en menor medida, por diversos invertebrados, aunque el espectro trófico de Anas platyrhyncos es considerablemente más amplio que el de las otras dos especies. A pesar de las diferencias tróficas existentes entre estas especies, en los tres casos se trata de aves acuáticas cuya densidad se ha visto favorecida, directa o indirectamente, por la alteración del cauce y de las márgenes de los cursos, por la modificación del régimen hidrológico e hidráulico y por la pérdida de calidad de las aguas superficiales.

El gran desarrollo de formaciones de Typha domingensis y Phragmites australis en la orilla aluvial del Tajo-Jarama, debido a la alteración del régimen hidrológico y al incremento de los niveles de nutrientes (MoLINA HOLGADO, 2003), incrementa la disponibilidad de recursos tróficos. Algunas especies, como Gallinula chloropus, consumen tallos, hojas, y en algunos casos semillas, de Lemna, Typha, Phragmites, Potamogeton, Rumex, 
Polygonum y Paspalum, entre otras especies (CRAMP, 1998; AMAT y SORIGER, 1984), plantas que en casi todos los casos son muy frecuentes en estos cursos debido a su alteración. Las plantas terrestres y palustres son menos importantes en la dieta de Fulica atra, pero entran en la misma otras especies como Cladofora y Spyrogira (CRAMP, 1998), algas verdes muy frecuentes en tramos fluviales contaminados como consecuencia del aumento de los niveles de fósforo (BORCHARDT, 1996; CUBILLO et AL., 1990), y otras algas como Vaucheria y Ectocarpus, o cianobacterias como Nostoc (CRAMP, 1998).

Otra especie dominante cuya alta densidad también parece estar en relación con la alteración la calidad de las aguas es Tachybaptus ruficollis, aunque la base de la dieta de este zampullín está formada por larvas e individuos adultos de varias especies de insectos (CRAMP, 1998).

Las aves limícolas también se benefician de la alteración de la calidad de las aguas superficiales, ya que en zonas mesosaprobias aumenta la proporción de algunos invertebrados, como los tubifícidos y Chironomus (AVILÉs y TORO, 1991; TORO, 1992), que forman parte de su dieta. GARCÍA DE JALÓN (1982) señala que el tramo bajo del río Jarama carece de vida macrobéntica. Según CUBILlO et AL. (1990) la diversidad y riqueza del macrobentos de los tramos más contaminados de los cursos de Madrid es muy baja, pero algunos oligoquetos como los tubifícidos o algunos dípteros como Chiromus gr. plumosus que viven en el fango y soportan mínimas concentraciones de oxígeno disuelto pueden llegar a alcanzar altas densidades. Por otra parte, la mejoría relativa de la calidad de las aguas del Tajo-Jarama tras la entrada en vigor del Plan de Saneamiento Integral de Madrid en la mitad de la década de los años 80 debe haber repercutido positivamente en las características del macrobentos de los tramos bajos del Tajo y del Jarama. PRIETO y GARCÍA DE JALÓN (1987) señalaron además la presencia de Physa fontonalis, Tubificidae, Corixidae, Notonectidae, Pericoma sp., Culcidae, Orthocladinae, Chiromus gr. plumosus, Deratopogonidae y Syrphidae en el río Jarama en San Martín de la Vega y en el Puente Largo, destacando en este último lugar por su elevada densidad Chironomus gr. plumosus.

La presencia de los invertebrados citados anteriormente explicaría la importancia del río Jarama para las aves limícolas durante los pasos migratorios y para algunas especies reproductoras como Himantopus himantopus, que alcanzan elevadas densidades en esta estación (139,8 aves/km en julio): la base de la dieta de estas aves está formada por insectos acuáticos, tanto en estado adulto como larvario, oligoquetos, crustáceos, moluscos, anélidos, etc. (CRAMP, 1998). Este autor señala en concreto la presencia de Tubifex tubifex en la dieta de Tringa ochropus, un oligoqueto presente en altas den- 
sidades en medios con elevados contenidos en nutrientes también consumido por Charadrius dubius. Para Himantopus himantopus indica en su dieta adultos y larvas de Coleoptera, Trichoptera, Hemiptera, Odonata, Diptera, Neuroptera, lepidoptera, crustáceos y moluscos (Physa, Succinea), arañas y anélidos. Las máximas densidades del díptero Chironomus gr. plumosus se producen en primavera-verano, igual que las de Himantopus himantopus, limícola cuya dieta en la ribera del Tajo-Jarama debe de estar constituida casi en exclusiva por estos dípteros.

\section{IV.3. Distribución, dominancia y constancia}

La evolución mensual de la distribución en función de la riqueza total mensual no muestra diferencias apreciables entre las tres zonas de estudio, salvo en el mes del enero para el Tajo-Ebro y en los meses de enero y febrero para el Ebro-Jarama. Estas diferencias expresan también la afinidad existente entre las comunidades de aves acuáticas del Tajo y del Jarama, y señalan las grandes diferencias que se producen entre las comunidades de aves acuáticas de estos dos cursos y la del río Ebro. Los elementos de amplia distribución del Ebro son principalmente especies ictiófagas, mientras que en el Tajo y en el Jarama estas especies son elementos incluidos en la categoría trófica materia vegetal-invertebrados.

La distribución de la riqueza total anual en las tres zonas de estudio no muestra variaciones significativas ni en función del grado de constancia de las especies $\left(\chi_{2}=1,2588 p=0,8683\right)$, aunque los elementos que forman el grupo de especies constantes varía entre cada ribera, principalmente entre el Ebro y el Tajo $(\mathrm{Ij}=0,27)$ y entre el Ebro y el Jarama $(\mathrm{Ij}=0,31)$, ya que la composición del grupo de especies dominantes en el Tajo y en el Jarama muestra una afinidad cualitativa elevada $(\mathrm{Ij}=0,61)$. Son rasgos de diferenciación entre las tres zonas de estudio la constancia de Phalacrocorax carbo, Circus aeroginosus y Alcedo atthis en el Ebro, y la constancia de Tachybaptus ruficollis, Anas strepera, Anas clypeata, Aythya ferina, Larus ridibundus y Actitis hypoleucos en el Tajo-Jarama. Al analizar la categoría trófica de estas especies se aprecia como los elementos constantes en la ribera del Ebro que no aparecen en el Tajo-Jarama son elementos ictiófagos, mientras que las especies que son elementos constantes en el Tajo-Jarama pero que no aparecen en el Ebro son mayoritariamente especies incluidas en la categoría trófica materia vegetal-invertebrados.

\section{CONCLUSIONES}

La estructura y composición de las comunidades de aves acuáticas de los ríos Tajo y Jarama muestran numerosos rasgos comunes, mientras que la 
comunidad de aves acuáticas del río Ebro se diferencia claramente de las anteriores. Son características comunes del Tajo y del Jarama, la elevada densidad y riqueza de las poblaciones de aves y su importancia como área de invernada para anátidas, fochas, garzas, cormoranes y gaviotas. Los valores de densidad y riqueza de la comunidad de aves acuáticas de la ribera del Ebro son siempre más bajos que en el Tajo y en el Jarama y los valores máximos de ambos parámetros se producen en los pasos migratorios.

Las comunidades de aves acuáticas de estos ríos no muestran diferencias apreciables ni en su estructura fenológica, ni en la distribución de los grados de constancia, ni tampoco en la distribución mensual de las categorías de distribución. La distribución de la riqueza por categorías de dominancia y de estructura trófica tampoco muestra diferencias apreciables. Sí son significativas las diferencias existentes en la composición y densidad de las categorías de dominancia y de las categorías tróficas, así como en los valores mensuales de diversidad $(\mathrm{H})$ y equitabilidad (J), más elevados siempre en el Ebro que en el Tajo y en el Jarama.

Las diferencias existentes entre las comunidades de aves acuáticas del Tajo-Jarama y el Ebro se relacionan con las características hidráulicas, hidrológicas y morfológicas de los cursos, así como con la calidad de las aguas de los mismos. La alteración del régimen hidrológico de los ríos Jarama y Tajo y la modificación de sus características hidráulicas, que ha favorecido la dominancia de los tramos de régimen lento frente a los de régimen rápido, repercute positivamente en el tamaño de la población invernante de aves acuáticas. La menor alteración hidrológica e hidráulica del río Ebro, que se manifiesta en el suceso frecuente de avenidas de recurrencia anual con puntas de caudal superiores a $2.000 \mathrm{~m}^{3} / \mathrm{s}$ y en la dominancia de los tramos de régimen rápido sobre los de régimen lento, inciden negativamente en la riqueza y densidad de la población de estas aves. La mala calidad de las aguas de los ríos Tajo y Jarama hace que la importancia relativa de las especies ictiófagas sea baja ante la falta de recursos tróficos, claramente menor que en el Ebro, donde estas especies son dominantes. Las especies incluidas en la categoría materia vegetal-invertebrados, como Anas platyrhynchos, Fulica atra y Gallinula chloropus, son los elementos dominantes en el Tajo y en el Jarama, beneficiadas por la alteración de la calidad de las aguas que provoca una alta disponibilidad de recursos tróficos (algas, algunas plantas nitrófilas, dípteros, oligoquetos, etc).

La morfología de la orilla aluvial también influye en la riqueza de las comunidades de aves. La existencia de extensos depósitos de gravas y arenas no fitoestabilizados en los ríos Ebro y Jarama se relaciona positivamente con el número de especies de aves limícolas presentes en estas riberas, mucho 
más escasas sin embargo en el Tajo, donde la extensión de este tipo de sedimentos fluviales es más reducida. La mayor importancia del río Jarama para este grupo de aves parece estar en relación con la presencia en densidades elevadas de algunos invertebrados característicos de comunidades propias de aguas fuertemente contaminadas como, por ejemplo, Chironomus gr. plumosus.

Cuadro 10. Abundancia y riqueza (evolución mensual río Jarama).

\begin{tabular}{|c|c|c|c|c|c|c|c|c|c|c|c|c|c|}
\hline & \multicolumn{11}{|c|}{1994} & \multicolumn{2}{|c|}{1995} \\
\hline & $F$ & $M$ & $A$ & $M$ & $J$ & $J l$ & $A$ & $S$ & $O$ & $N$ & $D$ & $E$ & $F$ \\
\hline Riqueza & 18 & 24 & 25 & 24 & 23 & 25 & 31 & 30 & 29 & 24 & 22 & 21 & 27 \\
\hline Kilómetros & 12,5 & 12,5 & 12,5 & 12,5 & 12,5 & 12,5 & 12,5 & 12,5 & 12,5 & 12,5 & 12,5 & 12,5 & 12,5 \\
\hline Tachybaptus rufficollis & 51 & 23 & 16 & 17 & 23 & 28 & 110 & 113 & 138 & 160 & 122 & 142 & 130 \\
\hline Podiceps nigricollis & & & & 1 & 4 & & 1 & & 1 & & & & \\
\hline Podiceps cristatus & & & & & & & & & 1 & & & & \\
\hline Phalacrocorax carbo & 2 & 2 & & & & & & & 4 & 9 & 26 & 9 & 10 \\
\hline Ixobrichus minutus & & & & & 1 & & 1 & & & & & & \\
\hline Nyctycorax nyctycorax & & & 30 & & 4 & 3 & 5 & & & & & & \\
\hline Bubulcus ibis & & 2 & 210 & & 11 & 1 & 30 & 4 & 6 & 7 & & & \\
\hline Egretta garzetta & & 2 & 1 & & 2 & - & 1 & 4 & 11 & 4 & 2 & & 2 \\
\hline Ardea cinerea & 3 & 2 & 4 & 1 & 2 & 2 & & 55 & 109 & 16 & 23 & 24 & 25 \\
\hline Ciconia ciconia & & & & & 1 & & 2 & & & & 5 & & 2 \\
\hline Anser anser & & & & & & & & & & & & & 1 \\
\hline Anas penelope & & & & & & & & 2 & & 1 & & 2 & \\
\hline Anas strepera & 8 & 30 & 32 & 10 & 1 & 1 & 5 & 15 & 87 & 129 & 50 & 37 & 87 \\
\hline Anas crecca & 19 & 12 & & & & & & 129 & 148 & 17 & 31 & 42 & 71 \\
\hline Anas platyrhynchos & 17 & 125 & 200 & 287 & 53 & 85 & 46 & 196 & 360 & 303 & 325 & 350 & 386 \\
\hline Anas acuta & & & 2 & & & & & 4 & 14 & 25 & 53 & 52 & 47 \\
\hline Anas querquedula & & 3 & 1 & & & & & 1 & & & & & \\
\hline Anas clypeata & 67 & 137 & 14 & & 5 & & 1 & 57 & 26 & 36 & 30 & 43 & 86 \\
\hline Netta rufina & & & & 2 & & & & & 1 & & & & \\
\hline Aythya ferina & & 2 & 47 & 49 & 65 & 135 & 43 & 21 & 22 & 14 & 27 & 4 & 24 \\
\hline Aythya fuligula & & & & 1 & & 1 & & & & & 2 & & 1 \\
\hline Oxyura leucocephala & & & & & & & & & 1 & & & & \\
\hline Oxyura jamaicensis & 1 & & & & & & & & & & & & \\
\hline Pandion haliaetus & & & & & & & & 2 & & & & & \\
\hline Circus ae & 2 & 2 & 3 & & & & & & 3 & 1 & 2 & & \\
\hline Rallus aquaticus & & & & & 1 & 1 & 1 & & 1 & 2 & & 1 & \\
\hline Porphyrio porphyrio & & & & & & & 1 & 1 & 2 & 2 & 3 & 3 & 2 \\
\hline Gallinula chloropus & 417 & 462 & 411 & 258 & 17 & 237 & 711 & 606 & 826 & 661 & 675 & 977 & 721 \\
\hline Fulica atra & 137 & 123 & 82 & 86 & 140 & 212 & 199 & 221 & 316 & 238 & 329 & 301 & 220 \\
\hline Himantopus himantopus & & 3 & 53 & 148 & 398 & 1.748 & 403 & 5 & 5 & & & & 1 \\
\hline Recurvirostra avoseta & & & & & 1 & & & & & & & & \\
\hline Burhinus oedicnemus & & & 5 & & & 1 & 3 & & & & & & 2 \\
\hline Charadrius hiaticula & & & & 10 & & & 5 & & & & & & \\
\hline Charadrius alexandrinus & & & 1 & & & & & & & & & & \\
\hline Charadrius dubius & & 1 & 18 & 13 & 24 & 52 & 158 & 4 & & 1 & & & 3 \\
\hline Pluvialis squatarola & & & & 3 & & & & & & & & & \\
\hline Vanellus vanellus & 75 & 15 & 6 & 3 & 94 & 47 & 81 & 2 & 55 & 218 & 72 & 17 & 120 \\
\hline Calidris alba & 2 & & & 1 & & & & & & & & & \\
\hline Calidris minuta & & & & 2 & & & 17 & & & & & & \\
\hline Calidris alpina & 4 & & 1 & 7 & & 4 & 2 & 3 & & & & & \\
\hline Gallinago gallinago & 23 & 16 & 6 & & & & 4 & 12 & 67 & 37 & 42 & 18 & 28 \\
\hline Limosa limosa & & & & & 2 & 16 & 1 & & & & & & \\
\hline Phylomachux pugnax & & 5 & 6 & 10 & & 4 & 21 & 10 & & & & & \\
\hline Tringa totanus & & 3 & & 9 & & 10 & 7 & 1 & 1 & & & & \\
\hline Tringa erythropus & & & & & & & & 1 & 1 & & & & \\
\hline Tringa nebularia & & & & 2 & & & 3 & 2 & & 1 & 1 & & 2 \\
\hline Tringra ochropus & 11 & 11 & 13 & & 37 & 64 & 79 & 22 & 18 & 27 & 21 & 14 & 29 \\
\hline Tringa glareola & & & & 1 & & 2 & 1 & & 1 & & & & \\
\hline Actitis hypoleuca & 2 & 1 & 3 & 30 & 1 & 67 & 148 & 13 & 3 & 8 & & 3 & 9 \\
\hline
\end{tabular}


Larus ridibundus

Larus fuscus

Gelochelidon nilotica

Childonias niger

Alcedo atthis

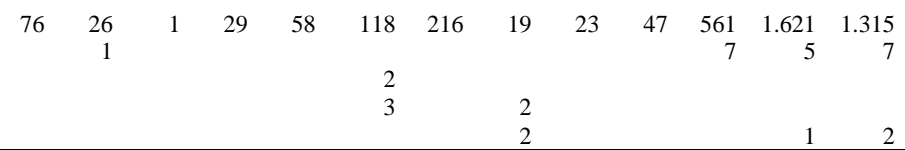

Cuadro 11. Abundancia y riqueza (evolución mensual río Tajo).

\begin{tabular}{|c|c|c|c|c|c|c|c|c|c|c|c|c|c|}
\hline & \multicolumn{11}{|c|}{1994} & \multicolumn{2}{|c|}{1995} \\
\hline & $F$ & $M$ & $A$ & $M$ & $J$ & $J l$ & $A$ & $S$ & $O$ & $N$ & $D$ & $E$ & $F$ \\
\hline Riqueza total & 21 & 20 & 28 & 20 & 18 & 22 & 24 & 18 & 24 & 25 & 23 & 25 & 24 \\
\hline Kilómetros & 18,3 & 20,3 & 20,3 & 20,3 & 20,3 & 20,3 & 20,3 & 20,3 & 20,3 & 20,3 & 20,3 & 49,9 & 20,3 \\
\hline Tachybaptus ruficollis & 75 & 73 & 58 & 24 & 51 & 62 & 80 & 59 & 113 & 63 & 83 & 137 & 81 \\
\hline Podiceps cristatus & & & & & & & & 1 & 3 & 1 & & & \\
\hline Phalacrocorax carbo & 82 & 153 & 2 & & & & & 4 & 112 & 187 & 229 & 364 & 205 \\
\hline Ixobrichus minutus & & & & & & & 4 & & & & & & \\
\hline Nycticorax nycticorax & 16 & 8 & 3 & 10 & 5 & 8 & 16 & 4 & 5 & 4 & 13 & 16 & 7 \\
\hline Bubulcus ibis & & & 2 & 6 & 3 & 23 & 26 & & 433 & & 24 & 94 & \\
\hline Egretta garzetta & 6 & 2 & 1 & 1 & & 2 & 12 & 14 & 10 & 3 & 6 & 13 & 9 \\
\hline Ardea cinerea & 55 & 23 & 2 & & 3 & 7 & 21 & 47 & 79 & 59 & 58 & 101 & 54 \\
\hline Ardea purpurea & & & 1 & & 1 & 4 & 5 & & & & & & \\
\hline Ciconia ciconia & & & & 2 & 1 & 2 & 3 & & & & & 2 & 1 \\
\hline Anas penelope & 6 & & & & 1 & 1 & & & 1 & 21 & 27 & & 24 \\
\hline Anas strepera & 95 & 78 & 14 & 16 & 24 & 25 & 27 & 17 & 35 & 31 & 127 & 85 & 63 \\
\hline Anas crecca & 77 & 16 & & & & & 7 & 4 & 8 & 82 & 63 & 69 & 83 \\
\hline Anas platyrhynchos & 1.185 & 831 & 902 & 644 & 442 & 861 & 703 & 892 & 1.540 & 2.009 & 1.510 & 2.723 & 747 \\
\hline Anas acuta & 4 & & & & & & & & & 1 & 2 & 3 & 7 \\
\hline Anas querquedula & & & 4 & & & & & & & & & & \\
\hline Anas clypeata & 214 & 135 & 9 & 1 & 2 & & 10 & 5 & 13 & 6 & 108 & 269 & 167 \\
\hline Netta rufina & & & 1 & & & & & & 1 & 1 & & & 1 \\
\hline Aythya ferina & 224 & 145 & 32 & 48 & 71 & 54 & 49 & 2 & 7 & 111 & 214 & 150 & 149 \\
\hline Aythya fuligula & 7 & 10 & 3 & 2 & 6 & 2 & 2 & & 2 & 5 & 49 & 27 & 7 \\
\hline Circus aeruginosus & 3 & 2 & 1 & 1 & & 2 & & & 1 & 1 & 2 & 7 & \\
\hline Rallus aquaticus & & & & & & & & & & 1 & & 1 & \\
\hline Porphyrio porphyrio & & & & & & & & & & & & 1 & \\
\hline Gallinula chloropus & 103 & 343 & 535 & 176 & 301 & 508 & 1.335 & 312 & 495 & 343 & 461 & 1.341 & 530 \\
\hline Fulica atra & 729 & 369 & 303 & 285 & 524 & 737 & 768 & 768 & 988 & 1.010 & 1.009 & 1.836 & 758 \\
\hline $\begin{array}{l}\text { Himantopus himan- } \\
\text { topus }\end{array}$ & & & 122 & 51 & 134 & 157 & 87 & 7 & & & 1 & & \\
\hline Recurvirostra avoseta & & & 1 & & & & & & & & & & \\
\hline Burhinus oedicnemus & & & & & & 1 & & & & & & & \\
\hline Charadrius dubius & & & 54 & 12 & 45 & 92 & 75 & 3 & 1 & & & & 3 \\
\hline Vanellus vanellus & 6 & & 1 & 11 & 7 & 62 & 6 & & 61 & 46 & & 18 & 18 \\
\hline Calidris minuta & & & & & & & 1 & & & & & & \\
\hline Calidris alpina & & & 1 & & & 1 & & & & & & & \\
\hline Gallinago gallinago & 3 & 5 & 4 & & & & 1 & & 2 & 7 & 6 & 25 & 16 \\
\hline Limosa limosa & & 1 & & & & & & & & & & & \\
\hline Philomachus pugnax & & & 6 & & & & & & & & & & \\
\hline Tringa totanus & & & 1 & 1 & & & & & & & & & \\
\hline Tringa ochropus & 1 & 1 & 24 & & 19 & 67 & 16 & 12 & 7 & 5 & 2 & 15 & 8 \\
\hline Actitis hypoleucos & & & 12 & 26 & & 106 & 62 & 7 & 10 & 1 & 3 & 8 & 2 \\
\hline Larus ridibundus & 66 & 8 & 1 & & & & 19 & 24 & & 28 & 115 & 152 & 130 \\
\hline Larus fuscus & 10 & 4 & & & & & & & & 5 & 10 & 21 & 21 \\
\hline Gelochelidon nilotica & & & & 1 & & & & & & & & & \\
\hline Childonias niger & & & & 2 & & & & & & & & & \\
\hline Alcedo atthis & & & & & & 1 & 1 & 1 & 1 & & & & \\
\hline
\end{tabular}

Cuadro 12. Abundancia y riqueza (evolución mensual río Ebro).

\begin{tabular}{|c|c|c|c|c|c|c|c|c|c|c|c|c|c|}
\hline & \multicolumn{4}{|c|}{1995} & \multicolumn{9}{|c|}{1996} \\
\hline & $S$ & $O$ & $N$ & $D$ & $E$ & $F$ & $M$ & $A$ & $M$ & $J$ & $J l$ & $A$ & $S$ \\
\hline Riqueza & 23 & 19 & 15 & 18 & 13 & 14 & 19 & 27 & 13 & 11 & 19 & 20 & 21 \\
\hline Kilómetros & 18,0 & 24,6 & 23,2 & 23,2 & 22,4 & 23,2 & 22,4 & 23,2 & 22,4 & 22,4 & 16,0 & 22,4 & 22,4 \\
\hline
\end{tabular}




\begin{tabular}{|c|c|c|c|c|c|c|c|c|c|c|c|c|c|}
\hline Tachybaptus rufficollis & 1 & 1 & & 1 & 1 & 2 & 3 & & & & & 1 & 1 \\
\hline Podiceps nigricollis & & & & 1 & & & & & & & & & \\
\hline Podiceps cristatus & & & & & & & 1 & 1 & & 1 & 2 & 3 & 2 \\
\hline Phalacrocorax carbo & 7 & 44 & 95 & 186 & 68 & 140 & 120 & 27 & & & 1 & & 28 \\
\hline Nycticorax nycticorax & & & & & & & & 2 & 9 & 1 & 14 & 18 & 5 \\
\hline Bubulcus ibis & & 6 & & & & & & & & & & & \\
\hline Egretta garzetta & 2 & & & 2 & & & & 1 & 1 & 6 & 14 & 13 & 7 \\
\hline Ardea cinerea & 86 & 57 & 75 & 41 & 19 & 25 & 11 & 11 & 7 & 31 & 39 & 101 & 11 \\
\hline Ardea purpurea & 4 & & & & & & & 8 & 8 & 12 & 7 & 10 & 31 \\
\hline Ciconia ciconia & 4 & 3 & 1 & 1 & 1 & & 3 & & 2 & 8 & & 5 & 7 \\
\hline Anser anser & & & & & 1 & & & & & & & & \\
\hline Anas penelope & & & & 4 & & & & & & & & & \\
\hline Anas strepera & & & & 6 & 4 & 2 & & & & & & & \\
\hline Anas crecca & 1 & 16 & & 5 & & 4 & 5 & & & & & 1 & 9 \\
\hline Anas platyrhynchos & 94 & 169 & 151 & 57 & 7 & 17 & 79 & 80 & 76 & 34 & 49 & 214 & 322 \\
\hline Anas acuta & & 2 & 4 & & & & & & & & & & \\
\hline Anas querquedula & & & & & & & & 1 & & & & & \\
\hline Anas clypeata & 1 & & & & & & & 3 & & & & & \\
\hline Pandion haliaetus & & & & & & & 1 & 2 & & & & & 2 \\
\hline Circus aeruginosus & 9 & 2 & 5 & 1 & 1 & 1 & 1 & 2 & & & & 2 & 4 \\
\hline Rallus aquaticus & 5 & & & & & & & & & & & & \\
\hline Gallinula chloropus & 9 & 8 & 4 & 10 & 6 & 8 & 17 & 11 & 3 & & 4 & 5 & 12 \\
\hline Fulica atra & 15 & 8 & 2 & 17 & 23 & 17 & 8 & 5 & & 1 & 2 & 6 & 1 \\
\hline Himantopus himantopus & & & & & & & & 13 & 1 & & 11 & 3 & \\
\hline Burhinus oedicnemus & & & & & & & & 2 & & & & & \\
\hline Charadrius dubius & 92 & 31 & 9 & & & & 59 & 155 & 64 & 14 & 87 & 80 & 68 \\
\hline Vanellus vanellus & 211 & 12 & 5 & & & & 13 & 1 & 1 & & 9 & & \\
\hline Calidris minuta & 13 & 12 & & & & & & & & & & & \\
\hline Calidris alpina & 1 & 5 & & 1 & & & & & 1 & & & & 2 \\
\hline Gallinago gallinago & 3 & 160 & 176 & 61 & 1 & 3 & 9 & 4 & & & & 2 & 2 \\
\hline Philomachus pugnax & & & & & & & & 4 & & & 3 & 1 & \\
\hline Tringa totanus & & & & & & & 3 & 8 & 1 & & 2 & & \\
\hline Tringa erythropus & 1 & & & & & & & 1 & & & & & \\
\hline Tringa glareola & 14 & & & & & & & 4 & & & 2 & & 7 \\
\hline Tringa ochropus & 68 & 55 & 67 & 15 & 2 & 3 & 14 & 23 & & 5 & 14 & 32 & 71 \\
\hline Actitis hypoleuca & 53 & 17 & 12 & 14 & 1 & 5 & 4 & 18 & 50 & 47 & 58 & 42 & 39 \\
\hline Larus ridibundus & & & 3 & & & & 3 & 3 & & & 6 & 10 & \\
\hline Larus fuscus & & & & & & 1 & & & & & & & \\
\hline Alcedo atthis & 11 & 7 & 8 & 2 & & 2 & 2 & 1 & & & 2 & 3 & 8 \\
\hline
\end{tabular}

\section{BIBLIOGRAFÍA}

Alegre, J.; FernándeZ, F.; HernándeZ, A.; SÁnCHEZ, A.J. (1987): «Estudio ecológico de las comunidades de aves invernantes en los parques leoneses». Ecología, $\mathrm{n}^{\mathrm{o}}$ 1: 211-223.

AMAT, J.A.; FERRER, X. (1988): «Respuesta de los patos invernantes en España a diferentes condiciones ambientales». Ardeola, $\mathrm{n}^{\circ} 35$ (1): 59-70.

AmAt, J.A.; Soriguer, R.C. (1984): «Alimentación invernal de la Polla de agua (Gallinula chloropus) en las Marismas del Guadalquivir». Ardeola, no 31: 136140.

Arratibel, P.; DeÁn-Pinedo, J.; Llamas, A.; MARTínez, O. (1995): Anuario ornitológico de Navarra, 1993-1994. Sociedad de Ciencias Naturales GOROSTI. Pamplona.

ArratiBel, P.; DeÁn-Pinedo, J.; Llamas,A. y Martínez, O. (1996): Anuario ornitológico de Navarra. Vol. 2. 1995. Sociedad de Ciencias Naturales GOROSTI. Pamplona. 
Arthington, A. H.; NAiman, R.J.; Mcclain, M.E.; Nilsson, C. (2010): «Preserving the biodiversity and ecological services of rivers: new challenges and research opportunities». Freshwater Biology, no 55: 1-16.

AvilÉs, J.; TORO, M. (1991): «Indicadores biológicos de la calidad de las aguas. Aplicación en la cuenca del Tajo». Ingeniería Civil, $\mathrm{n}^{\circ}$ 80: 23-28.

Bergarandi, A.; Arzoz, M.J.; CAMPos, F.; LeKuOnA, J.M. (1995): «Aumento de la población reproductora de Garza Imperial (Ardea purpurea) en Navarra, Norte de España». Ardeola, no 42(2): 201-203.

BIRLIFE INTERNATIONAL (2004): Birds in Europe: population estimates, trends and conservation status. Cambridge. BirdLife International.

Blanco, G.; Acha, A.; Cuevas, J.A.; Rodríguez, P. y Velasco, T. (1995). «Fenología de la reproducción y productividad de anátidas en ríos del valle medio del Tajo». Ardeola, no 43: 31-39.

Blanco, G.; Velasco,T.; GriJalbo,J.; Ollero, J. (1994): «Great Cormorantt Settlement of a New Winttering area in Spain». Colonial Waterbirds, $\mathrm{n}^{\circ}$ 7: 173-180.

BlanCo,G.; GómeZ, F.; Morato, J. (1995): «Composición de la dieta y tamaño de la presa de Cormorán Grande (Phalacrocorax carbo sinensis) durante su invernada en los ríos y graveras del Centro de España». Ardeola, no 42 (2): 125-131.

Boldreghini, P.; Casani, L.; Montavari, R.; SAntolini, R.; TAnarelli, R (1993): «The population of the Great Cormorant wintering in the Po Delta River during 1988-1989» En Status and conservation of see birds (Aguilar, Mombailliu \& Paterson, eds.). Madrid. SEO. 369-370.

Boldreghini, P.; Pandolfi, M.; SAntolini, R. (1993): «The winter diet of the Great Cormorant on the Po Delta River (preliminary data)» En Status and conservation of see birds (Aguilar, Mombailliu \& Paterson, eds.). Madrid. SEO: 357-359.

BorChARDT, M. (1996): «Nutrients» en Algal Ecology. FreshwaterBentic Ecoystems (Stevenson, Bothwell \& Lowe, eds.). New York. Academic Press. 183-227.

BRAUZE, T. (2004): «Species diversity of breeding waterbirds of oxbow lakes on the flood terrace of the Vistula river between Ciechocinek and Solec Kujawski», Zoologica Poloniae, no 49 (1-4): 211-218.

CALLEJA, J.A. (2009): «92A0. Alamedas, olmedas y saucedas de las regiones atlántica, alpina, mediterránea macaronésica» en Bases ecológicas preliminares para la conservación de los tipos de hábitats de interés comunitario en España (VV.AA.). Madrid. Ministerio de Medio Ambiente, Medio Rural y Marino.

CAMPOS, F.; LEKUONA, J.M. (1994): «La población de Cormorán grande (Phalacrocorax carbo) en el Norte de España y Suroeste de Francia». Ardeola, $n^{o} 41$ (1): $13-18$.

CANO, J. (2005): «Densidad de algunas especies de aves comunes en un tramo del río Manzanares» en Anuario Ornitológico de Madrid 2004 (DE LA PUENTE, J.; PÉREZ-Tris, J.; BERMEJO, A.; JUAN, M., eds.). Madrid. SEO-Monticola, 80-95.

CRAMP, S. (1998): The birds of the Western Paleartic on CD-Rom". Oxford. Oxford University Press.

Cubillo, F.; CASAdo, C.; Castillo, V. (1990): Caudales ecológicos. Estudio de regímenes de caudales mínimos en los cauces de la Comunidad de Madrid. Madrid. Agencia de Medio Ambiente de la Comunidad de Madrid. 
CuEVAS, J.A. 1997. «Estudio de una comunidad reproductora de aves acuáticas en un curso fluvial fuertemente antropizado». En MANRIQUE, J.; SÁNCHEZ, A.; SuÁreZ, F. y YANES, M. (Eds). Actas de las XII Jornadas Ornitológicas Españolas. Añmería. Instituto de Estudios Almerienses. Diputación de Almería. 4754

Cuevas, J.A.; Acha, A.; Blanco, G.; De Miguel, J.M.; Delgado, J.A.; Ruiz, P. y VELASCO, T. 2000. «Estudio de los patrones temporales de la comunidad de aves acuáticas presente en un ecosistema fluvial». I Congreso Ibérico de Ecología. Santiago de Compostela, Septiembre de 2000).

Cuevas, J.A.; Acha, A.; Blanco, G.; Ruiz, P.; Velasco, T.; Delgado, J.A. y De Miguel, J.A. 2000. Biodiversidad en ecosistemas fluviales: las aves acuáticas en la cuenca media del Tajo. Serie Documentos, $n^{\circ}$ 31. Madrid. Centro de Investigaciones Ambientales de la Comunidad de Madrid Fernando González Bernáldez.

De la Puente, J.; Ponce, C. (2004): «Censos invernales de Cormorán grande (Phalacrocorax carbo) en la Comunidad de Madrid. Invernadas 2001-2001 y 20022003» en Anuario Ornitológico de Madrid 2002 (De la Puente, J.; Pérez-Tris, J.; Bermejo, A., eds): Madrid. SEO-Monticola: 126-133.

Debout, G. (1987): «Le Grand Cormoran, Phalacrocorax carbo carbo, en France: le populations nicheurs littorales». Alauda, $\mathrm{n}^{\circ}$ 55: 35-45.

Del Moral, J. (2000): «Censos de aves acuáticas invernantes en la Comunidad de Madrid. 1998-1999» en Anuario Ornitológico de Madrid 2000 (BERMEJO, A.; DE LA Puente, J.; SEOANE, J.; eds.). Madrid. SEO-Monticola: 160-167.

Del Moral, J. (2001): «Censos de aves acuáticas invernantes en la Comunidad de Madrid. 1999-2000» en Anuario Ornitológico de Madrid 2002 (BERMEJO, A.; DE LA Puente, J.; SEOANE, J.; eds.). Madrid. SEO-Monticola: 142-149.

Del Moral, J. (2002): «Censos de aves acuáticas invernantes en la Comunidad de Madrid. 2000-2001» en Anuario Ornitológico de Madrid 2001 (BERMEJO, A.; DE LA Puente, J.; SeOANE, J.; eds.). Madrid. SEO-Monticola: 138-145.

Del Moral, J. (2003): «Censos de aves acuáticas invernantes en la Comunidad de Madrid. 2001-2002» en Anuario Ornitológico de Madrid 2002 (DE LA PUENTE, J.; PÉREZ-TRIS, J.; BERMEJO, A.; eds.). Madrid. SEO-Monticola:118-125.

GALÁN E. (2004): «Sequías climáticas en la Meseta meridional» en Historia, clima y paisaje : estudios geográficos en memoria del profesor Antonio López Gómez. Valencia. Universidad de Valencia: 337-352.

GÁMEZ, I. (1994): «Población y evolución demográfica de las aves acuáticas de La Rioja». Zubía, no 6: 207-304.

GARCÍA DE JALÓN, D. (1982): «Los insectos como bioindicadores de la contaminación». Quercus, $\mathrm{n}^{\circ}$ 4: 36-39.

GREGERSEN, J. (1991): «The development of the Danis Comorant population 198088 and some comments on the breeding succes» en Workshop 1989 on Cormorants (EERDEN, M.R. y ZILJSTRA, M. eds.). Lelystad. Rijkswaterstraat Directorate Flevoland.. 36-38.

HeRnÁNDEZ, A. y Velasco, T. (1990): «Dinámica estacional de la comunidad de limícolas en el río Bernesga (Meseta Norte, España)». Ecología, no 4: 229-233. 
HuBÁLEK, Z. 1999: «Seasonal changes of bird communities in a managed lowland riverine ecosystem». Folia Zoologica, $\mathrm{n}^{\circ}$ 48: 203-210.

KoffijberG, K.; Bauer, H.; Boschert, M.; Delacour, G.; Dronneau, C.; KELLER, V.; SUDFELDT, C. (2001): Waterbirds in the Rhine Valley in 1999/2000. With a summary of trends in 1980-2000. Institute for Inland Water Management and Waste Water Treatment (RIZA). Lelystad.

LEIVA, A; ARAGONÉS, J.; CARRASCO, M. (1989): «Invernada de no paseriformes en el río Guadalquivir a su paso por la ciudad de Córdoba, 1987-1988», Boletín del Parque Nacional del Delta del Ebro, no 4: 33-36.

LEKUONA, J.M. y CAMPOS, J. (1996): «Diferencia en la alimentación del Cormorán Grande (Phalacrocoraz carbo) entre la ría de Bidasoa y su estuario». Ardeola, $\mathrm{n}^{\circ}$ 43 (2): 109-205.

MARTí, R.; DEL MORAL, J.C. (Eds.) (2003): La invernada de aves acuáticas en España. Madrid. Ministerio de Medio Ambiente.

MARTín, J.A.; PÉREZ, A. (1990): «Movimiento del Martín pescador (Alcedo atthis) en España». Ardeola, no 37 (1): 13-18.

MASON, C. y MACDONALD, S.M. 2000: «Numbers of wintering waterbirds on rivers in eastern England». Wildfowl, $\mathrm{n}^{\circ}$ 51: 215-219.

MAson, C.F.; HofMANN, T.A.; MACDONALD, S.M. (2006): «The winter bird community of river corridors in eastern England in relation to habitat variables». Ornis Fennica 83:73-85.

MEAdOws, B.S. (1972): «Kingfisher numbers and stream polution». Ibis,110: 443.

Molina Holgado, P. (2003). Análisis y comparación de la vegetación de las riberas de los ríos Ebro, Tajo y Jarama. Madrid. Universidad Autónoma de Madrid, Obra Social Cajamadrid.

Molina Holgado, P.; Berrocal, A.B. (2006): «Los efectos ambientales de la regulación de los cursos de la cabecera de la cuenca del Tajo: la reducción de los bosques aluviales del Tajo-Jarama» en III Congreso de Ingeniería Civil, territorio y Medio Ambiente. Madrid. Colegio de Ingenieros de Caminos, Canales y Puertos.

MolinA, B. (2004): «Censos de aves acuáticas invernantes en la Comunidad de Madrid. Invernada 2002-2003» en Anuario Ornitológico de Madrid 2004 (DE LA Puente, J.; Pérez-Tris, J.; Bermejo, A.; JuAN, M. eds.). Madrid. SEOMonticola, 114-121.

MolinA, B. (2005): «Censos de aves acuáticas invernantes en la Comunidad de Madrid. Invernada 2003-2004» en Anuario Ornitológico de Madrid 2004 (DE LA Puente, J.; PÉrez-Tris, J.; Bermejo, A.; JuAn, M. eds.). Madrid. SEOMonticola, 152-159.

MolinA, B. (2006): «Censos de aves acuáticas invernantes en la Comunidad de Madrid. Invernada 2004-2005» en Anuario Ornitológico de Madrid 2005 (DE LA Puente, J.; PÉrez-Tris, J.; Bermejo, A.; JuAn, M. eds.). Madrid. SEOMonticola, 118-129.

MolinA, B. (2007): «Censos de aves acuáticas invernantes en la Comunidad de Madrid» en Anuario Ornitológico de Madrid 2006 (DE LA PUENTE, J.; PÉREZ-TRIS, J.; JUAN, M.; BERMEJO, A.; eds.). Madrid. SEO-Monticola, 80-91. 
MolinA, F.; GARCíA, A.; RodríGUEZ, M. (1987): «El nuevo cauce del Guadaira como espacio de interés para las aves acuáticas». Oxyura, nº 4: 45-60.

Moreno-OPO, R. (2009): «Censo de cormorán grande Phalacrocorax carbo invernante en la provincia de Toledo. Enero 2003» en Anuario Ornitológico de Toledo (SÁnCHEZ, J.F., ed.). Toledo. Esparvel. 132-139.

NuMMI, P.; ELMBERG,J. y SJÖBERG, K. (1994): «Habitat distribution of the mallard in relation to vegetation structure, food, and population density». Hidrobiologia, n²78/280: 247-252.

PRIETO, G.; GARCÍA DE JALÓN, D. (1987): «Evolución temporal de la calidad biológica de las aguas del río Jarama». Montes, Revista de Ámbito Forestal, 17: 3040.

REBANE, WALICZKY y TURNER (1997): «Boreal and temperate forests» en Habitats for birs in Europe: a conservation strategy for de wider Enviromet (TUCKER, G.M. y Evans, M.I. -coords.-), pp. 203-238 . BirLife Conservation Series No. 6. Cambridge. BirLife International,.

Round, P.D.; Moss, M. (1984): «The waterbird populations of three Welsh rivers». Bird Study no 31: 61-68.

Røv, N. (1991): «Recent population development and breeding succes of Great Cormorant in Norway». en Workshop 1989 on Cormorants (EERDEN,M.R. y ZILJSTRA, M. eds.), Lelystad. Rijkswaterstraat Directorate Flevoland. 27-29.

SEO-ARAGón (1994): Anuario ornitológico de Aragón 1991-1992. Zaragoza. SEOAragón.

SEO-ARAGÓN (1997): Anuario ornitológico de Aragón 1993-1994. Zaragoza. SEOAragón.

SEO-BIRDLIFE, 1997. «Censos de aves acuáticas invernantes en la Comunidad de Madrid. Enero 1996» en Anuario Ornitológico de Madrid 1996 (DE LA PUENTE, J.; BERMEJO, A.; SEOANE, J.. eds.). Madrid. SEO-Monticola, 72-75.

SEO-BIRDLIFE, 1998. «Censos de aves acuáticas invernantes en la Comunidad de Madrid. Enerp 1997» en Anuario Ornitológico de Madrid 1997 (DE LA PUENTE, J.; BERMEJO, A.; SEOANE, J.. eds.). Madrid. SEO-Monticola, 90-95.

SEO-BIRDLIFE, 1999. «Censos de aves acuáticas invernantes en la Comunidad de Madrid. Invernada 1998-1999» en Anuario Ornitológico de Madrid 1998 (DE LA Puente, J.; BermeJo, A.;SEOANE, J.. eds.). Madrid. SEO-Monticola, 138-143.

Stevens, L.E.; BuCK, K.A.; Brown, .T.; Kline, N.C. (1997): «Dam and geomorphological influences on Colorado river waterbird distribution, Grand Canyon, Arizona, USA». Regulted Rivers: Research \& Management, $\mathrm{n}^{\circ} 13: 151-169$

TELlERÍA, J.L. (1986): Manual para el censo de Vertebrados terrestres. Madrid. Raíces.

TORO, M. (1992): «Relaciones ecológicas de los macroinvertabrados bentónicos en ríos y su papel como indicadores biológicos». Ingeniería Civil, 86: 79-102.

TORO, M.; RoBles, S.; TEJERO, I. (2009): «3280. Ríos mediterráneos de caudal permanente del Paspalo-Agrostidion con cortinas ribereñas de Salix y Populus alba» en Bases ecológicas preliminares para la conservación de los tipos de hábitats de interés comunitario en España (VV.AA.). Madrid. Ministerio de Medio Ambiente, Medio Rural y Marino. 
Velasco, T. y Alberto, L.J. (1993). «Number, main localities, and distribution maps, of waders wintering in Spain». Wader Study Group Bull. 70: 33-41.

VELASCO, T. (1992):«Waders along the rivers in Spain». Wader Study Group Bull., $n^{\circ} 64: 41-44$.

Velasco, T. (2009). «Un ejemplo de la importancia ornitológica de las vegas de encharcamiento temporal en La Mancha: el Pozo de la Cambronera (Alcázar de San Juan, Ciudad Real)» en Anuario Ornitológico de Ciudad Real 2006-2007 (CASAS, F.; ARREDONDO, A.; LÓPEZ-JAMAR, J.; Eds.).Ciudad Real. SEO-Ciudad Real.

Velasco, T.; Blanco, G. (1996): «Descripción de la comunidad invernal de aves en un parque urbano». Airo, 7 (1): 26-35.

ZIJLSTRA. M.; EERDEN, M.R. (1991): «Development and breeding population of Great Cormorants Phalacrocorax carbo in the Netherlands til 1989». En Workshop 1989 on Cormorants (EERDEN,M.R. \& ZILJSTRA, M. eds.). Lelystad. Rijkswaterstraat Directorate Flevoland. 53-60.

ZIMMERMAN, H.; RUTSCHKE, E. (1991): «Population and population development of the Great Cormorant Phalacorocorax carbo sinensis in the German Democratic Republic» en Workshop 1989 on Cormorants (EERDEN,M.R. y ZILJSTRA, M. eds.). Lelystad. Rijkswaterstraat Directorate Flevoland. 45-48. 